\title{
Lagged Covariance and Cross-Covariance Operators of Processes in Cartesian Products of Abstract Hilbert Spaces
}

\author{
Sebastian Kühnert*
}

\begin{abstract}
A major task in Functional Time Series Analysis is measuring the dependence within and between processes, for which lagged covariance and cross-covariance operators have proven to be a practical tool in well-established spaces. This article deduces estimators and asymptotic upper bounds of the estimation errors for lagged covariance and cross-covariance operators of processes in Cartesian products of abstract Hilbert spaces for fixed and increasing lag and Cartesian powers. We allow the processes to be non-centered, and to have values in different spaces when investigating the dependence between processes. Also, we discuss features of estimators for the principle components of our covariance operators.
\end{abstract}

MSC 2010 subject classifications: 47B10, 60G05, 62J10

Keywords: Estimation; functional time series; lag- $h$-covariance operator; lag- $h$-cross covariance operator; principle components

\section{Introduction}

Functional Data Analysis (FDA) and Functional Time Series Analysis (FTSA), the research areas dealing with (time series/processes of) random functions, have gained more and more significance, since considering random functions instead of vectors, provided the context allows it, assures more accurate results. Such an extension on infinite-dimensional spaces is enabled by ongoing developments in processing techniques, and unproblematic for separable Banach spaces from a mathematical point of view, see Ledoux \& Talagrand [31]. FDA/FTSA find applications in various fields. In economics, to predict the stock market trend [23] and the value at risk with intra-day return curves [37], comparing yield curves [42], and energy forecasts [9], [10]. In medicine, for characterizing COVID-19 data [45] (U.S. data) and [4] (Italy data), and analyzing brain images [46] and dementia [6]. For predicting mortality rates [13]. In face [35] and speech recognition [15]. For forecasting pollution concentrations [3] and forest biodiversity [32]. Also, Functional Principal Component Analysis (FPCA) was used to examine rainfall variability [17] and flight data [24]. For extensive introductions to FDA/FTSA, see Ferraty \& Vieu [12], Ramsay \& Silverman [38], Bosq [5], Horváth \& Kokoszka [19] and Hsing \& Eubank [22].

In FTSA, the analysis of the dependence structure within and between given processes is of great importance. If these are wide-sense stationary (often denoted as weak/second-order stationary), where for convenience usually (strictly) stationarity and finite second moments are assumed, this can be done by using lag-h-covariance operators and lag-h-cross-covariance operators, respectively. The lag $h$ thereby denotes the time difference of interest. Another important subject of study in FTSA is FPCA, since functional principle components, i.e., the eigenvalues and eigenfunctions of the lag-0-covariance operator, the covariance operator of a stationary process, yield an efficient representation.

*EMAIL: s.kuehnert_math@gmx.de 


\subsection{State of the art}

Probabilistic features of and estimators for lag- $h$-covariance operators $\mathscr{C}_{\mathbf{X} ; h}$ of stationary processes $\boldsymbol{X}=$ $\left(X_{k}\right)_{k \in \mathbb{Z}}$ with values in $L^{2}[0,1]$, the space of measurable, square-Lebesgue integrable real valued functions with domain $[0,1]$, are widely studied for fixed lag $h$, see, e.g., [5], [19], [22], [34], [27]. Further, [39] developed covariance estimators in the space of continuous functions $C[0,1]$, [48] in tensor product Sobolev-Hilbert spaces, [33] for continuous surfaces, and [18], [1] for arbitrary separable Hilbert spaces. [34], [39], [18], [1] constrained their assertions to autoregressive (AR) processes, where [1] deduced the results for a random AR(1) operator. Thereby, [5], [19], [22], [1] utilized classical moment estimators, [27] estimated the integral kernels, in [18], [34] truncated spectral decompositions occured having estimated principle components, and [48] used operator regularized covariance estimators. Also, the limit distribution of the estimation errors of the lag-0-covariance operators was discussed in [26], [28].

FPCA in the Hilbert space $L^{2}[0,1]$ is extensively discussed in the existing literature, both from a probabilistic and statistical point of view. In [5], [19], [22], [26], [28] one finds asymptotic upper bounds for the principle components, both estimated seperately and uniformly, in sense of convergence in the second mean as well as almost surely. Moreover, [49] introduced $L^{1}$-norm FPCA.

A comprehensive study of lag-h-cross-covariance operators $\mathscr{C}_{\boldsymbol{X}, \boldsymbol{Y} ; h}$ of stationary $L^{2}[0,1]$-valued processes $\boldsymbol{X}=\left(X_{k}\right)_{k \in \mathbb{Z}}, \boldsymbol{Y}=\left(Y_{k}\right)_{k \in \mathbb{Z}}$ can be found in Rice \& Shum [36]. They established operator estimates, discussed methods measuring their significance and deduced their limit distribution. Aue \& Klepsch [2], who intensively discussed the estimation procedure of operators of linear, invertible processes in $L^{2}[0,1]$, had to estimate lag- $h$-cross-covariance operators of specific processes having values in Cartesian products of $L^{2}[0,1]$ in order derive their main results. Enabling processes to have values in Cartesian products is also handy when studying $\operatorname{AR}(p)$ processes with $p>1$, see [5]. Also, the quite recent work of Sarkar \& Panaretos [41] dealt in great detail with covariance estimation of functional data defined over multidimensional domains.

\subsection{Our contributions}

This article studies lagged covariance and cross-covariance operators of stationary processes in Cartesian products of abstract Hilbert spaces based on ideas in [36] and [2]. The focus is on deducing moment estimators and asymptotic upper bounds of the estimation errors for these operators. This is also done for the principle components of lag-0-covariance operators. They are estimated individually, and uniformly in sense of a supremum of a set of indices whose cardinality depends on a certain rate of convergence. Particularly worth mentioning is that this work's results facilitate a high degree of flexibility. This is because all processes are allowed to attain values in arbitrary Cartesian products of separable Hilbert spaces. Further, when the objects of investigation are lagged cross-covariance operators between two processes, we allow them to attain values in different spaces. Moreover, all results are stated for processes with arbitrary first moments, and the lag $h$ as well as the processes' Cartesian powers are allowed to be fixed or increase w.r.t. the sample sizes. In the works [29] resp. [30], slightly milder results were utilized to derive asymptotic upper bounds of estimation errors for operators of $(\mathrm{G}) \mathrm{ARCH}$ and linear, invertible processes, and the principle components of lag-0-covariance operators.

Investors of, e.g., solar and other power stocks of European companies could ask themselves what impact monthly sunshine duration in central Europe, see Fig 1, will have on their share values one month ahead, see Fig 2. This can be analyzed by using our lag-1-cross-covariance operators, and our lag-h-covariance operators might be advantageous for understanding the dependence structure within the processes in Fig 1-2. 

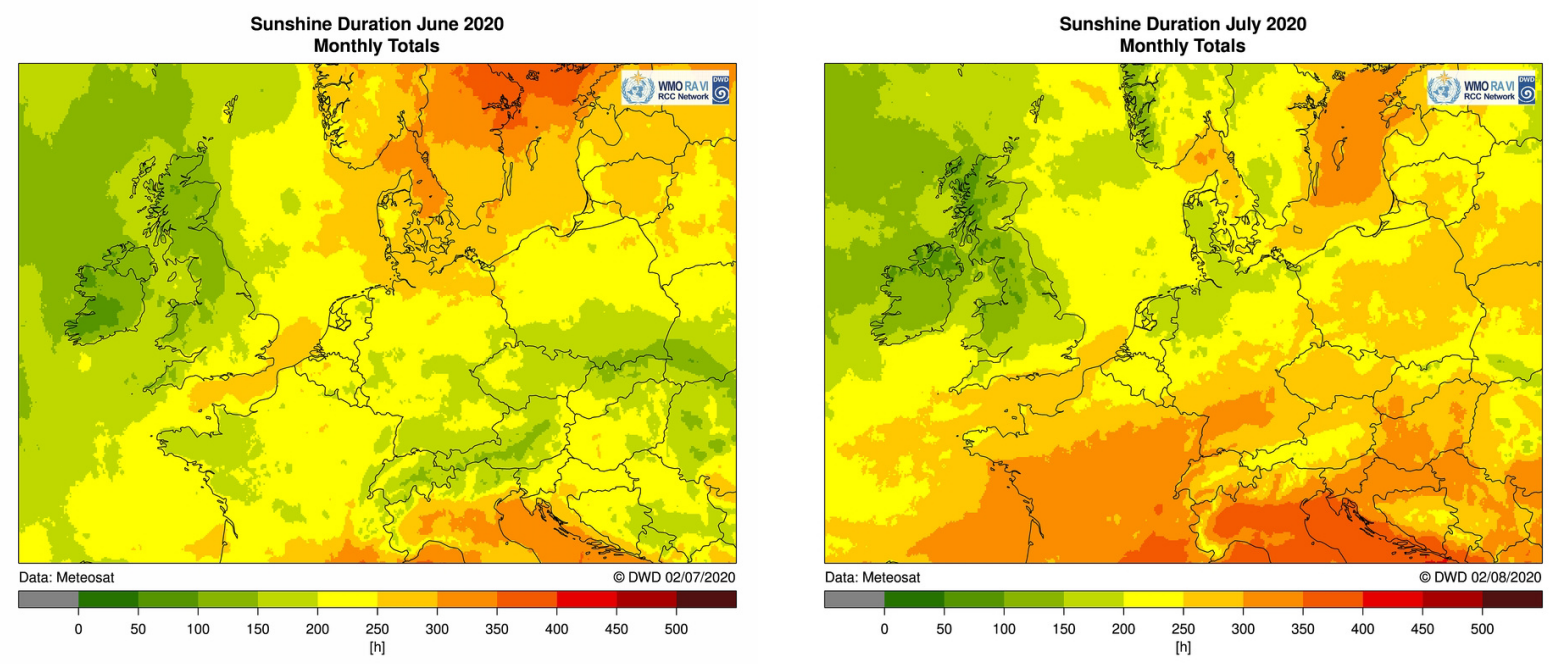

Figure 1: Graphs of monthly sunshine duration in central europe in June and July 2020, interpretable as two consecutive realizations of an $L^{2}[0,1]^{2}$-valued process, from the homepage www.dwd.de of the German Meterological Service.
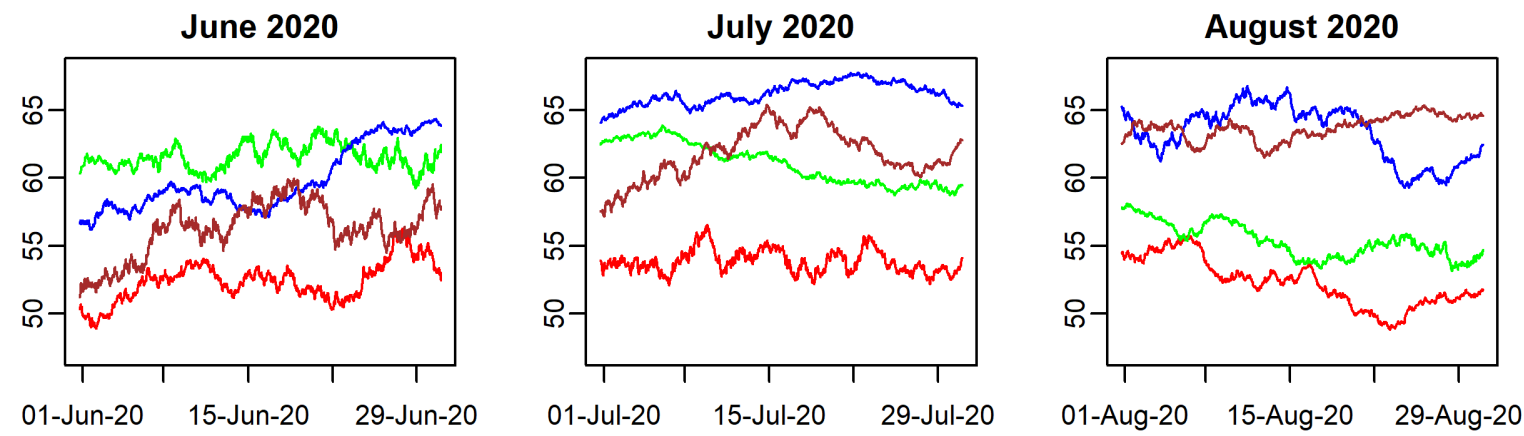

Figure 2: Three consecutive realizations of a fictitious process, which is identifiable as an $\left(L^{2}[0,1]\right)^{4}$-process, describing the share values of four assets of an portfolio, e.g., measured in EUR. The step width used is $\frac{1}{1000}$.

\subsection{Outline}

The rest of this paper is organized as follows. Section 2 outlines our notation, restates important terminology, definitions and interrelationships of several operator types, defines our (lagged) (cross-)covariance operators and studies their probabilistic features, and briefly explains $L^{p}-m$-approximibility. Section 3 introduces our estimators for the lagged (cross-)covariance operators and for the principle components of the lag-0-covariance operator, and derives asymptotic upper bounds of the estimation errors. Section 4 conducts a simulation study. Section 5 summarizes the main results and outlines future research. Moreover, Section 6 contains proofs. 


\section{Definitions and basics}

\subsection{Notation}

$\lfloor\cdot\rfloor$ denotes the floor function, $\operatorname{sgn}(\cdot)$ the sign function and $\mathbf{1}_{A}(\cdot)$ the indicator function of a set $A$. For sequences $\left(a_{n}\right)_{n \in \mathbb{N}},\left(b_{n}\right)_{n \in \mathbb{N}} \subseteq(0, \infty), a_{n} \sim b_{n}$ denotes $\frac{a_{n}}{b_{n}} \rightarrow 1, a_{n} \asymp b_{n}$ denotes $\frac{a_{n}}{b_{n}} \rightarrow c$ for some $c \neq 0$, (for $n \rightarrow \infty$ ) $a_{n}=\omega\left(b_{n}\right)$ if $b_{n}=\mathrm{o}\left(a_{n}\right)$ and $a_{n}=\Omega\left(b_{n}\right)$ if $b_{n}=\mathrm{O}\left(a_{n}\right)$ with common asymptotic notation $\mathrm{o}(\cdot), \mathrm{O}(\cdot)$, and $\Xi\left[a_{n}, b_{n}\right):=\Omega\left(a_{n}\right) \cap \mathrm{o}\left(b_{n}\right), \Xi\left[a_{n}, b_{n}\right]:=\Omega\left(a_{n}\right) \cap \mathrm{O}\left(b_{n}\right)$. Also, $0_{V}$ stands for the identity element of addition of a vector space $V, \mathbb{I}_{V}: V \rightarrow V$ for the identity operator, and operator for a linear map. Hereinafter, let $\left(\mathcal{H},\langle\cdot, \cdot\rangle_{\mathcal{H}}\right)$ be a real, separable Hilbert space. On Hilbert spaces we assume the norms to be induced by their inner product, and abbreviate 'complete orthonormal system' by $C O N S$. For $x, y \in \mathcal{H}, x \perp y$ denotes $\langle x, y\rangle_{\mathcal{H}}=0$. We define scalar multiplication and vector addition on $\mathcal{H}^{n}:=\left\{\left(x_{1}, \ldots, x_{n}\right)^{T} \mid x_{1}, \ldots, x_{n} \in \mathcal{H}\right\}$, with $n \in \mathbb{N}$, componentwise. Then, $\left(\mathcal{H}^{n},\langle\cdot, \cdot\rangle_{\mathcal{H}^{n}}\right)$ where $\langle\boldsymbol{x}, \boldsymbol{y}\rangle_{\mathcal{H}^{n}}:=\sum_{i=1}^{n}\left\langle x_{i}, y_{i}\right\rangle_{\mathcal{H}}$ for $\boldsymbol{x}:=\left(x_{1}, \ldots, x_{n}\right)^{T}, \boldsymbol{y}:=\left(y_{1}, \ldots, y_{n}\right)^{T} \in \mathcal{H}^{n}$, is a real, separable Hilbert space. In all respects, our random elements are defined on some common probability space $(\Omega, \mathfrak{A}, \mathbb{P})$. $X \stackrel{d}{=} Y$ means two random variables $X, Y$ are equal in distribution, and 'a.s.' denotes almost surely. For processes $\left(X_{n}\right)_{n}$ and $\left(Y_{n}\right)_{n}, X_{n}=\mathrm{O}_{\mathbb{P}}\left(Y_{n}\right)$ (for $\left.n \rightarrow \infty\right)$ means $\left(X_{n} / Y_{n}\right)_{n}$ is asymptotically P-stochastic bounded. For $p \in[1, \infty), L_{\mathcal{H}}^{p}=L_{\mathcal{H}}^{p}(\Omega, \mathfrak{A}, \mathbb{P})$ is the space of (classes of) $\mathcal{H}$-valued random variables $X$ with $\nu_{p, \mathcal{H}}(X):=\left(\mathbb{E}\|X\|_{\mathcal{H}}^{p}\right)^{1 / p}<\infty$, a process $\left(X_{k}\right)_{k \in \mathbb{Z}}$ of $\mathcal{H}$-valued random variables is an $L_{\mathcal{H}}^{p}$-process if $X_{k} \in L_{\mathcal{H}}^{p}$ for all $k$, and centered if $\mathbb{E}\left(X_{k}\right)=0_{\mathcal{H}}$ for all $k$ with expectation in Bochner-integral sense, see [22], p. 40-45.

\subsection{Some basic operator theory}

Now, we state important spaces of operators between real, separable Hilbert spaces $\left(\mathcal{H}_{i},\langle\cdot, \cdot\rangle_{\mathcal{H}_{i}}\right)$ for $i=1,2$. For thorough overviews of operators between Hilbert spaces, see the monographs Dunford \& Schwartz [11], Gohberg et al. [14], Weidmann [47].

The space of bounded operators mapping from $\mathcal{H}_{1}$ to $\mathcal{H}_{2}$ will be denoted by $\mathcal{L}_{\mathcal{H}_{1}, \mathcal{H}_{2}}$, with $\mathcal{L}_{\mathcal{H}_{1}}:=$ $\mathcal{L}_{\mathcal{H}_{1}, \mathcal{H}_{1}}$, where an operator $A: \mathcal{H}_{1} \rightarrow \mathcal{H}_{2}$ is bounded if

$$
\|A\|_{\mathcal{L}_{\mathcal{H}_{1}, \mathcal{H}_{2}}}:=\sup _{\|x\|_{\mathcal{H}_{1} \leq 1}}\|A(x)\|_{\mathcal{H}_{2}}<\infty
$$

Such operators are continuous, and $\left(\mathcal{L}_{\mathcal{H}_{1}, \mathcal{H}_{2}},\|\cdot\|_{\mathcal{L}_{\mathcal{H}_{1}, \mathcal{H}_{2}}}\right)$ is a Banach space. We denote the subspace of finite-rank operators of $\mathcal{L}_{\mathcal{H}_{1}, \mathcal{H}_{2}}$ by $\mathcal{F}_{\mathcal{H}_{1}, \mathcal{H}_{2}}$, with $\mathcal{F}_{\mathcal{H}_{1}}:=\mathcal{F}_{\mathcal{H}_{1}, \mathcal{H}_{1}}$. Further, $A^{*}$ denotes the adjoint of $A \in \mathcal{L}_{\mathcal{H}_{1}, \mathcal{H}_{2}}$, where $A^{*} \in \mathcal{L}_{\mathcal{H}_{2}, \mathcal{H}_{1}}$.

A crucial subspace of $\mathcal{L}_{\mathcal{H}_{1}, \mathcal{H}_{2}}$ is the space of compact operators mapping from $\mathcal{H}_{1}$ to $\mathcal{H}_{2}$, where $A \in \mathcal{L}_{\mathcal{H}_{1}, \mathcal{H}_{2}}$ is compact if $A$ maps the unit ball of $\mathcal{H}_{1}$ to a compact set in $\mathcal{H}_{2}$. Such operators possess the singular value decomposition

$$
A=\sum_{j=1}^{\infty} s_{j}\left(e_{j} \otimes f_{j}\right)
$$

with $x \otimes y:=\langle x, \cdot\rangle_{\mathcal{H}_{1}} y$ for $x \in \mathcal{H}_{1}, y \in \mathcal{H}_{2}$, where $\left(e_{j}\right)_{j \in \mathbb{N}}$ and $\left(f_{j}\right)_{j \in \mathbb{N}}$ are CONS of $\mathcal{H}_{1}$ and $\mathcal{H}_{2}$, respectively, and where $\left(s_{j}\right)_{j \in \mathbb{N}}$ is the monotonically decreasing zero sequence of non-negative numbers of $A$ which are called singular values. The decay rate of the singular values of a compact operator $A \in \mathcal{L}_{\mathcal{H}_{1}, \mathcal{H}_{2}}$ can be interpreted as a regularity measure of $A$, and expressed by the $p$-Schatten-norm

$$
\|A\|_{p}:=\left(\sum_{j=1}^{\infty} s_{j}^{p}\right)^{1 / p}, \quad p \in[1, \infty),
$$


where the inequality $\|A\|_{p} \leq\|A\|_{q}$ holds for all $p<q \cdot\left(\mathscr{S}_{\mathcal{H}_{1}, \mathcal{H}_{2}}^{p},\|\cdot\| \|_{p}\right)$ is a Banach space for all $p \in[1, \infty)$, where the $p$-Schatten-class is defined by

$$
\mathscr{S}_{\mathcal{H}_{1}, \mathcal{H}_{2}}^{p}:=\left\{A \in \mathcal{L}_{\mathcal{H}_{1}, \mathcal{H}_{2}} \mid\|A\|_{p}<\infty\right\},
$$

with $\mathscr{S}_{\mathcal{H}_{1}, \mathcal{H}_{2}}^{p} \subsetneq \mathscr{S}_{\mathcal{H}_{1}, \mathcal{H}_{2}}^{q}$ for all $p<q$. The essential classes are $\mathcal{N}_{\mathcal{H}_{1}, \mathcal{H}_{2}}:=\mathscr{S}_{\mathcal{H}_{1}, \mathcal{H}_{2}}^{1}$ with $\mathcal{N}_{\mathcal{H}_{1}}:=\mathcal{N}_{\mathcal{H}_{1}, \mathcal{H}_{1}}$ $\|\cdot\|_{\mathcal{N}_{\mathcal{H}_{1}, \mathcal{H}_{2}}}:=\|\cdot\|_{1}$, and $\mathcal{S}_{\mathcal{H}_{1}, \mathcal{H}_{2}}:=\mathscr{S}_{\mathcal{H}_{1}, \mathcal{H}_{2}}^{2}$ with $\mathcal{S}_{\mathcal{H}_{1}}:=\mathcal{S}_{\mathcal{H}_{1}, \mathcal{H}_{1}},\|\cdot\|_{\mathcal{S}_{\mathcal{H}_{1}, \mathcal{H}_{2}}}:=\|\cdot\|_{2}$, the spaces of nuclear/trace class resp. Hilbert-Schmidt operators. The trace of $A \in \mathcal{N}_{\mathcal{H}_{1}}$ is $\operatorname{defined} \operatorname{by} \operatorname{tr}(A):=$ $\sum_{j=1}^{\infty}\left\langle A\left(e_{j}\right), e_{j}\right\rangle_{\mathcal{H}_{1}}$, and $\left(\mathcal{S}_{\mathcal{H}_{1}, \mathcal{H}_{2}},\langle\cdot, \cdot\rangle_{\mathcal{S}_{\mathcal{H}_{1}, \mathcal{H}_{2}}}\right)$ is a separable Hilbert space, where

$$
\langle A, B\rangle_{\mathcal{S}_{\mathcal{H}_{1}, \mathcal{H}_{2}}}:=\sum_{j=1}^{\infty}\left\langle A\left(e_{j}\right), B\left(e_{j}\right)\right\rangle_{\mathcal{H}_{2}}, \quad A, B \in \mathcal{S}_{\mathcal{H}_{1}, \mathcal{H}_{2}},
$$

and where $\left(e_{j}\right)_{j \in \mathbb{N}}$ is an arbitrary CONS of $\mathcal{H}_{1}$ in both definitions.

On $\mathcal{H}_{1}:=L^{2}[0,1]$, an integral operator $A$ mapping from $\mathcal{H}_{1}$ to $\mathcal{H}_{1}$ is defined by the Lebesgue integral

$$
(A(x))(t):=\int_{0}^{1} a(s, t) x(s) \mathrm{d} s, \quad x \in \mathcal{H}_{1}, t \in[0,1]
$$

if it exists, where $a:[0,1]^{2} \rightarrow \mathbb{R}$ is a measurable function, the (integral) kernel of $A$. Such an operator satisfies $A \in \mathcal{S}_{\mathcal{H}_{1}}$ iff $\int_{0}^{1} \int_{0}^{1} a^{2}(s, t) \mathrm{d} s \mathrm{~d} t<\infty$.

\subsection{Features of our operators}

Here, we define (cross-)covariance operators and their lagged versions on real, separable Hilbert spaces, and outline some of their features (for a definition and features of these operators on Banach spaces, see [5]). Thereby, $\left(\mathcal{H}_{i},\langle\cdot, \cdot\rangle_{\mathcal{H}_{i}}\right)$ denote real, separable Hilbert spaces for $i=1,2$.

Definition 2.1. Let $X, Y$ be $L_{\mathcal{H}_{1}}^{2}$ resp. $L_{\mathcal{H}_{2}}^{2}{ }^{-v a l u e d}$ random variables, and let $m_{X}:=\mathbb{E}(X), m_{Y}:=\mathbb{E}(Y)$. Then, the covariance operator of $X$ is defined by

$$
\mathscr{C}_{X}:=\mathbb{E}\left(\left(X-m_{X}\right) \otimes\left(X-m_{X}\right)\right)
$$

and the cross-covariance operator of $X, Y$ is defined by

$$
\mathscr{C}_{X, Y}:=\mathbb{E}\left(\left(X-m_{X}\right) \otimes\left(Y-m_{Y}\right)\right) .
$$

For centered random variables $X, Y$, which is no restriction if $X, Y$ are integrable since $X^{\prime}:=X-\mathbb{E}(X)$ and $Y^{\prime}:=Y-\mathbb{E}(Y)$ are centered in any case, covariance and cross-covariance operators possess the following main probabilistic features.

Lemma 2.1. Let $X, Y$ be centered $L_{\mathcal{H}_{1}}^{2}$ resp. $L_{\mathcal{H}_{2}}^{2}$-valued random variables.

(a) $\mathscr{C}_{X}$ is a self-adjoint, positive semi-definite operator with $\mathscr{C}_{X} \in \mathcal{N}_{\mathcal{H}_{1}}$ and

$$
\left\|\mathscr{C}_{X}\right\|_{\mathcal{N}_{\mathcal{H}_{1}}}=\mathbb{E}\|X\|_{\mathcal{H}_{1}}^{2}
$$

and $\mathscr{C}_{X}$ possesses the representation

$$
\mathscr{C}_{X}=\sum_{j=1}^{\infty} c_{j}\left(\mathfrak{c}_{j} \otimes \mathfrak{c}_{j}\right)
$$

where $\left(c_{j}\right)_{j \in \mathbb{N}}$ is the eigenvalue sequence which is w.l.o.g. monotonically decreasing, non-negative and absolutely-summable, and where $\left(\mathfrak{c}_{j}\right)_{j \in \mathbb{N}}$ is the related eigenfunction sequence of $\mathscr{C}_{X}$ which is a CONS of $\mathcal{H}_{1}$. 
(b) $\mathscr{C}_{X, Y} \in \mathcal{N}_{\mathcal{H}_{1}, \mathcal{H}_{2},} \mathscr{C}_{X, Y}^{*}=\mathscr{C}_{Y, X} \in \mathcal{N}_{\mathcal{H}_{2}, \mathcal{H}_{1}}$ and

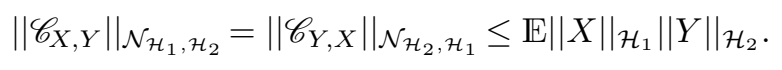

Furthermore,

$$
\text { independence of } X, Y \Rightarrow \mathscr{C}_{X, Y}=0_{\mathcal{L}_{\mathcal{H}_{1}, \mathcal{H}_{2}}},
$$

and if $\mathcal{H}_{1}=\mathcal{H}_{2}, \mathscr{C}_{X, Y}=0_{\mathcal{L}_{\mathcal{H}_{1}}}$ implies $\mathbb{E}\langle X, Y\rangle_{\mathcal{H}_{1}}=0$.

(c) If $\mathcal{H}_{1}=\mathcal{H}_{2}=L^{2}[0,1], \mathscr{C}_{X}$ and $\mathscr{C}_{X, Y}$ are integral operators with kernels $k_{X}(s, t):=\operatorname{Cov}(X(s), X(t))$ resp. $k_{X, Y}(s, t):=\operatorname{Cov}(X(s), Y(t)), s, t \in[0,1]$.

Similarly to covariances and variances for real-valued random variables hold the following calculation rules for (cross-)covariance operators.

Lemma 2.2. Let $W, X$ resp. $Y, Z$ be centered $L_{\mathcal{H}_{1}}^{2}$ resp. $L_{\mathcal{H}_{2}}^{2}$-valued random variables. Also, let $A \in \mathcal{L}_{\mathcal{H}_{1}}$ and $B \in \mathcal{L}_{\mathcal{H}_{2}}$.

(a) The covariance operator satisfies

$$
\mathscr{C}_{W+X}=\mathscr{C}_{W}+\mathscr{C}_{W, X}+\mathscr{C}_{X, W}+\mathscr{C}_{X}
$$

where $\mathscr{C}_{W+X}=\mathscr{C}_{W}+\mathscr{C}_{X}$ if $W, X$ are independent. Further, for the cross-covariance operator holds

$$
\mathscr{C}_{W+X, Y+Z}=\mathscr{C}_{W, Y}+\mathscr{C}_{W, Z}+\mathscr{C}_{X, Y}+\mathscr{C}_{X, Z}
$$

(b) The covariance operator of the $L_{\mathcal{H}_{1}}^{2}$-valued random variable $A(X)$ satisfies

$$
\mathscr{C}_{A(X)}=A \mathscr{C}_{X} A^{*}
$$

and for the cross-covariance operator of the $L_{\mathcal{H}_{1}}^{2}$ resp. $L_{\mathcal{H}_{2}}^{2}$-valued variables $A(X)$ and $B(Y)$ holds

$$
\mathscr{C}_{A(X), B(Y)}=B \mathscr{C}_{X, Y} A^{*}
$$

For the definition of the functional counterparts of the auto-covariance and cross-covariance function of real-valued processes, which are the lag- $h$-covariance resp. lag- $h$-cross-covariance operators, given processes do not necessarily have to be strictly, but wide-sense stationary. To reiterate, a process $\left(X_{k}\right)_{k}$ is (strictly) stationary if $\left(X_{k_{1}+h}, \ldots, X_{k_{n}+h}\right) \stackrel{d}{=}\left(X_{k_{1}}, \ldots, X_{k_{n}}\right)$ holds for all $k_{1}, \ldots, k_{n}, h$ and $n \in \mathbb{N}$, and an $L_{\mathcal{H}_{1}}^{2}$-process $\boldsymbol{X}:=\left(X_{k}\right)_{k}$ is wide-sense stationary if for all $k$ holds $\mathbb{E}\left(X_{k}\right)=c$ for some $c \in \mathcal{H}_{1}$ and if for all $k, l$ holds $\mathscr{C}_{X_{k}, X_{l}}=\mathscr{C}_{X_{0}, X_{l-k}}$.

Definition 2.2. Let $\boldsymbol{X}:=\left(X_{k}\right)_{k \in \mathbb{Z}}, \boldsymbol{Y}:=\left(Y_{k}\right)_{k \in \mathbb{Z}}$ be wide-sense stationary $L_{\mathcal{H}_{1}}^{2}$ resp. $L_{\mathcal{H}_{2}}^{2}$-processes. Then, the lag-h-covariance operator of $\boldsymbol{X}$ is defined by

$$
\mathscr{C}_{\boldsymbol{X} ; h}:=\mathscr{C}_{X_{0}, X_{h}}, \quad \forall h \in \mathbb{Z},
$$

with $\mathscr{C}_{\boldsymbol{X}}:=\mathscr{C}_{\boldsymbol{X} ; 0}$, and the lag-h-cross-covariance operator of $\boldsymbol{X}, \boldsymbol{Y}$ is defined by

$$
\mathscr{C}_{\boldsymbol{X}, \boldsymbol{Y} ; h}:=\mathscr{C}_{X_{0}, Y_{h}}, \quad \forall h \in \mathbb{Z}
$$

Remarks 2.1. (a) Lemmas 2.1-2.2 can be applied to lag- $h$-(cross-)covariance operators. Thus, $\mathscr{C}_{\boldsymbol{X} ; h}^{*}=$ $\mathscr{C}_{\boldsymbol{X} ;-h}$ and $\mathscr{C}_{\boldsymbol{X}, \boldsymbol{Y} ; h}^{*}=\mathscr{C}_{\boldsymbol{Y}, \boldsymbol{X} ;-h}$ for any $h$. Also, $\mathscr{C}_{\boldsymbol{X} ; h}=0_{\mathcal{L}_{\mathcal{H}_{1}}}$ for $h \neq 0$ if $\boldsymbol{X}:=\left(X_{k}\right)_{k \in \mathbb{Z}}$ consists of independent variables, and if $\boldsymbol{X}:=\left(X_{k}\right)_{k \in \mathbb{Z}}, \boldsymbol{Y}:=\left(Y_{k}\right)_{k \in \mathbb{Z}}$ are independent, $\mathscr{C}_{\boldsymbol{X}, \boldsymbol{Y} ; h}=0_{\mathcal{L}_{\mathcal{H}_{1}, \mathcal{H}_{2}}}$. 
(b) Since Lemma 2.1 (c) for (cross-)covariance can be transferred to lag- $h$-(cross-)covariance operators, it is reasonable that these operators contain the expression '(cross-)covariance' as well.

In the following, we illustrate a specific lag-0-covariance operator. For further, but somewhat more complicated examples and sketches, see Section 4.

Example 2.1. Let $\mathcal{H}:=L^{2}[0,1]$, and let $\varepsilon:=\left(\varepsilon_{k}\right)_{k \in \mathbb{Z}}$ be a process with

$$
\varepsilon_{k}(t):=\frac{Z_{k}+B_{k}(t)}{\sqrt{1+t}} \text { a.s., } \quad \forall k \in \mathbb{Z}, \forall t \in[0,1],
$$

where $Z_{k} \sim \mathcal{N}(0,1), \boldsymbol{B}_{\boldsymbol{k}}=\left(B_{k}(t)\right)_{t \in[0,1]}$ are Wiener processes, and where $\ldots, Z_{-1}, \boldsymbol{B}_{-\mathbf{1}}, Z_{0}, \boldsymbol{B}_{\mathbf{0}}, Z_{1}, \boldsymbol{B}_{\mathbf{1}}, \ldots$ are independent. Then, $\left(\varepsilon_{k}\right)_{k \in \mathbb{Z}}$ is an i.i.d., centered $L_{\mathcal{H}}^{4}$-process with $\varepsilon_{0}(t) \sim \mathcal{N}(0,1)$ for all $t \in[0,1]$, and for the integral kernel $k_{\varepsilon ; 0}=k_{\varepsilon}$ of $\mathscr{C}_{\varepsilon ; 0}=\mathscr{C}_{\varepsilon}$ holds

$$
k_{\varepsilon}(s, t)=\operatorname{Cov}\left(\varepsilon_{0}(s), \varepsilon_{0}(t)\right)=\sqrt{\frac{1+\min (s, t)}{1+\max (s, t)}}, \quad \forall s, t \in[0,1] .
$$

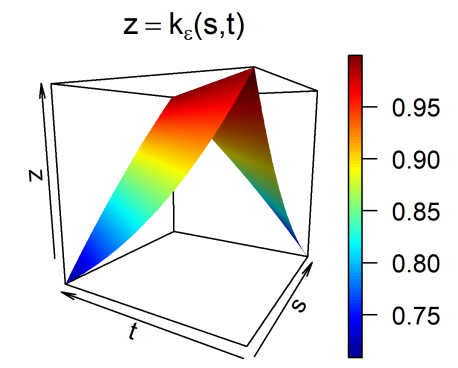

Figure 3: The integral kernel $k_{\varepsilon}(s, t)$ in $(2.10)$ for $s, t \in[0,1]$.

\section{$2.4 \quad L^{p}$-m-approximibility}

For deriving asymptotic upper bounds of estimation errors for operators or functionals related to a stationary process, usually weak dependence of the given process is needed. We impose $L^{p}$-m-approximibility, a well manageable measure of weak dependence developed by Hörmann \& Kokoszka [21].

Definition 2.3. Let $\left(\mathcal{H},\langle\cdot, \cdot\rangle_{\mathcal{H}}\right)$ be a separable Hilbert space and let $p \geq 1$. Then, a process $\left(Z_{k}\right)_{k \in \mathbb{Z}}$ is called $L_{\mathcal{H}}^{p}$-m-approximable if it is an $L_{\mathcal{H}}^{p}$-process with

$$
Z_{k}=f\left(\varepsilon_{k}, \varepsilon_{k-1}, \ldots\right)
$$

for any $k$, where $\left(\varepsilon_{k}\right)_{k \in \mathbb{Z}}$ is an i.i.d. process with values in a measurable space $S$ and where $f: S^{\infty} \rightarrow \mathcal{H}$ is a measurable function, such that

$$
\sum_{m=1}^{\infty} \nu_{p, \mathcal{H}}\left(Z_{m}-Z_{m ; m}\right)<\infty
$$

Thereby, $\nu_{p, \mathcal{H}}(\cdot):=\left(\mathbb{E}\|\cdot\|_{\mathcal{H}}^{p}\right)^{1 / p}$ and

$$
Z_{k ; m}:=f\left(\varepsilon_{k}, \varepsilon_{k-1}, \ldots, \varepsilon_{k-m+1}, \varepsilon_{k-m ; k}, \varepsilon_{k-m-1 ; k}, \ldots\right)
$$

where $\left(\varepsilon_{k ; n}\right)_{k}$ are independent copies of $\left(\varepsilon_{k}\right)_{k}$ for each $n$.

$L_{\mathcal{H}}^{p}-m$-approximibility of a process thus means it is non-anticipative w.r.t.another process, that is (2.11), and approximable by an $m$-dependent process so that the approximation errors measured by the

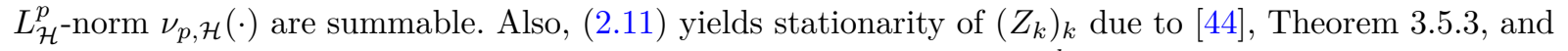
$\left(Z_{k ; m}\right)_{k}$ are stationary, $m$-dependent processes for each $m$ with $Z_{k ; m} \stackrel{d}{=} Z_{k}$ for all $k, m$. 


\section{Main results}

Herein, we discuss the main results of this paper, namely the estimation procedure for lag- $h$-covariance and lag- $h$-cross-covariance operators of $\mathcal{U}^{m}$ - resp. $\mathcal{V}^{n}$-valued processes for $m, n \in \mathbb{N}$, and for the principal components of lag-0-covariance operators. Thereby, $\left(\mathcal{U}^{m},\langle\cdot, \cdot\rangle_{\mathcal{U}^{m}}\right)$ and $\left(\mathcal{V}^{n},\langle\cdot, \cdot\rangle_{\mathcal{V}^{n}}\right)$ are real, separable Hilbert spaces coming from real, separable Hilbert spaces $\left(\mathcal{U},\langle\cdot, \cdot\rangle_{\mathcal{U}}\right)$ and $\left(\mathcal{V},\langle\cdot, \cdot\rangle_{\mathcal{V}}\right)$. Throughout this article, we assume that our processes have the following representations.

Assumption 3.1. (a) Let $\boldsymbol{X}:=\left(X_{k}\right)_{k \in \mathbb{Z}}$ be an $L_{\mathcal{U}}^{4}$-m-approximable process and let $\mathscr{X}:=\left(\mathscr{X}_{k}\right)_{k \in \mathbb{Z}}$ be an $\mathcal{U}^{m}$-valued process with

$$
\mathscr{X}_{m+j}:=\left(X_{m+j p}, \ldots, X_{1+j p}\right)^{T}
$$

for any $j \in \mathbb{Z}$ and some $p \in \mathbb{N}$. Further, $X_{1}, \ldots, X_{M}$ is a sample of $\boldsymbol{X}$ with $M \geq m$, thus $\mathscr{X}_{m}, \ldots, \mathscr{X}_{\tilde{\mathscr{M}}}$ with $\tilde{\mathscr{M}}=\tilde{\mathscr{M}}_{M}:=\left\lfloor\frac{M-m}{p}\right\rfloor+m$ is a sample of $\mathscr{X}$, and the sample size is $\mathscr{M}=\mathscr{M}_{M}:=\tilde{\mathscr{M}}_{M}-m+1$.

(b) Let $\boldsymbol{Y}:=\left(Y_{k}\right)_{k \in \mathbb{Z}}$ be an $L_{\mathcal{V}}^{4}$-m-approximable process and non-anticipative w.r.t.the same i.i.d. process $\left(\varepsilon_{k}\right)_{k}$ as $\boldsymbol{X}$ in (a), and let $\mathscr{Y}:=\left(\mathscr{Y}_{k}\right)_{k \in \mathbb{Z}}$ be an $\mathcal{V}^{n}$-valued process with

$$
\mathscr{Y}_{n+j}:=\left(Y_{n+j q}, \ldots, Y_{1+j q}\right)^{T}
$$

for any $j \in \mathbb{Z}$ and some $q \in \mathbb{N}$. Moreover, $Y_{1}, \ldots, Y_{N}$ with $N \geq n$ is a sample of $\boldsymbol{Y}$, thus $\mathscr{Y}_{n}, \ldots, \mathscr{Y}_{\tilde{N}}$ with $\tilde{\mathscr{N}}=\tilde{\mathscr{N}}_{N}:=\left\lfloor\frac{N-n}{q}\right\rfloor+n$ is a sample of $\mathscr{Y}$, and the sample size is $\mathscr{N}=\mathscr{N}_{N}:=\tilde{\mathscr{N}}_{N}-n+1$.

Remarks 3.1. (a) $\left(\mathscr{X}_{k}\right)_{k}$ and $\left(\mathscr{Y}_{k}\right)_{k}$ are stationary processes since the underlying processes $\left(X_{k}\right)_{k}$ resp. $\left(Y_{k}\right)_{k}$ are stationary due to $L^{4}-m$-approximibility.

(b) The common case in the assumption above is when $m=n=1$. This case can always be derived as a specific example from our results.

(c) Imposing two different sample sizes $M, N$ for $\left(X_{k}\right)_{k}$ resp. $\left(Y_{k}\right)_{k}$ is useful if more data of one process can be collected w.r.t. time, but one is not willing to relinquish information by choosing the minimum of $M, N$.

(d) Choosing $p, q$ so that $1 \leq p<m, 1 \leq q<n$, enables to reuse the first $(m-p)$ resp. $(n-q)$ entries of $\mathscr{X}_{k}$ resp. $\mathscr{Y}_{k}$ for the last $(m-p)$ resp. $(n-q)$ entries of $\mathscr{X}_{k+1}$ resp. $\mathscr{Y}_{k+1}$. If one needs to successively stack (realizations of) $X_{k}^{\prime} s$ resp. $Y_{k}^{\prime} s$ in a vector of length $m$ resp. $n$, one has to put $p=m, q=n$. One could also choose $p>m, q>n$, which is useful when data is missing, since by an appropriate choice of $p$ and $q$, the time indices where (realizations of) $X_{k}^{\prime} s$ and/or $Y_{k}^{\prime} s$ are missing can be bridged.

(e) $\left(\mathscr{X}_{k}\right)_{k}$ resp. $\left(\mathscr{Y}_{k}\right)_{k}$ is i.i.d. for $p \geq m$ resp. $q \geq n$ if $\left(X_{k}\right)_{k}$ resp. $\left(Y_{k}\right)_{k}$ is.

(f) Whenever large sample sizes of $\left(\mathscr{X}_{k}\right)_{k}$ and $\left(\mathscr{Y}_{k}\right)_{k}$ are needed, and reusing values of $\left(X_{k}\right)_{k}$ resp. $\left(Y_{k}\right)_{k}$ does not cause issues, $p$ and $q$ should be set as small as possible, to wit $p=q=1$. Thereby, processes $\left(\mathscr{X}_{k}\right)_{k}$ as in (3.1) with $p=1$ were used for estimating the operators of $L^{2}[0,1]$-valued AR in [5], (G)ARCH in [30], and invertible linear processes in [2], [30].

Our model also allows the vector lengths $m, n$ and the numbers describing the 'degree of reuse' $p, q$ of given variables to depend on the sample sizes as follows.

Assumption 3.2. (a) $\left(m_{M}\right)_{M},\left(p_{M}\right)_{M} \subseteq \mathbb{N}$ are sequences with $m=m_{M}, p=p_{M}=\Xi[1, M)=\Omega(1) \cap$ $\mathrm{o}(M)$ where $a_{n}=\Omega\left(b_{n}\right)$ means $b_{n}=\mathrm{O}\left(a_{n}\right)$.

(b) $\left(n_{N}\right)_{N},\left(q_{N}\right)_{N} \subseteq \mathbb{N}$ are sequences with $n=n_{N}, q=q_{N}=\Xi[1, N)$. 
From the Assumptions 3.1-3.2 (a) and Assumptions 3.1-3.2 (b) follows

$$
\begin{aligned}
\mathscr{M} & =\mathscr{M}_{M} \sim p^{-1} M, \\
\text { resp. } \quad \mathscr{N} & =\mathscr{N}_{N} \sim q^{-1} N .
\end{aligned}
$$

Since the time difference where some variable has a certain effect on another one could also change over time respectively the sample size, we also allow the lag $h \in \mathbb{Z}$ to vary w.r.t. given sample sizes as follows. Assumption 3.3. (a) $h=h_{M}=\Xi\left[1, p^{-1} M\right)$;

(b) $h=h_{N}=\Xi\left[1, q^{-1} N\right)$.

Lemma 3.1. Let Assumptions 3.1-3.2 (a) hold. Then,

$$
\hat{m}_{\mathscr{X}}:=\frac{1}{\mathscr{M}_{M}} \sum_{i=m}^{\tilde{\mathscr{M}}_{M}} \mathscr{X}_{i}
$$

is an unbiased estimator for the first moment $m_{\mathscr{X}}:=\mathbb{E}\left(\mathscr{X}_{1}\right)$, and for $M \rightarrow \infty$ :

$$
\mathbb{E}|| \hat{m}_{\mathscr{X}}-m_{\mathscr{X}} \|_{\mathcal{U}^{m}}^{2}=\mathrm{O}\left(m \mathscr{M}_{M}^{-1}\right)=\mathrm{O}\left(m p M^{-1}\right) .
$$

Remarks 3.2. Lemma 3.1 can be generalized to higher moments if the related power of the random variable of the process is well-defined on the given Hilbert space. Powers of random variables are for instance welldefined on $L^{2}[0,1]$. There, $X^{2}$ denotes the pointwise product of $X$ and $X, X^{3}$ e.g. the pointwise product of $X^{2}$ and $X, X^{4}$ e.g. the pointwise product of $X^{3}$ and $X$ etc.

\subsection{Estimation of lag- $h$-covariance operators}

When estimating lag- $h$-covariance operators, we distinguish, as for real-valued processes, between centered processes and those with an unknown first moment.

If the process $\boldsymbol{X}=\left(X_{k}\right)_{k}$ in Assumption 3.1 (a) is centered and consequently also $\mathscr{X}=\left(\mathscr{X}_{k}\right)_{k}$, we estimate $\mathscr{C}_{\mathscr{X} ; h}$ with $|h|<\mathscr{M}_{M}$ through

$$
\hat{\mathscr{C}}_{\mathscr{X} ; h}:= \begin{cases}\frac{1}{\mathscr{M}_{M, h}} \sum_{k=m+|h|}^{\tilde{\mathscr{M}}_{M}} \mathscr{X}_{k} \otimes \mathscr{X}_{k+h}, & h<0 \\ \frac{1}{\mathscr{M}_{M, h}} \sum_{k=m}^{\tilde{\mathscr{M}}_{M, h}} \mathscr{X}_{k} \otimes \mathscr{X}_{k+h}, & h \geq 0\end{cases}
$$

where $\mathscr{M}_{M, h}:=\mathscr{M}_{M}-|h|, \tilde{\mathscr{M}}_{M, h}:=\tilde{\mathscr{M}}_{M}-|h|$. The operators $\hat{\mathscr{C}}_{\mathscr{X} ; h}$ are finite-rank operators $\left(\hat{\mathscr{C}}_{\mathscr{X} ; h} \in \mathcal{F}_{\mathcal{U}^{m}}\right)$ with $\hat{\mathscr{C}}_{\mathscr{X} ; h}=\hat{\mathscr{C}}_{\mathscr{X}} ;-h$, and $\hat{\mathscr{C}}_{\mathscr{X}}:=\hat{\mathscr{C}}_{\mathscr{X} ; 0}$ is self-adjoint and positive semi-definite.

Theorem 3.1. Let Assumptions 3.1-3.3 (a) hold and let $\boldsymbol{X}$ be centered. Then, $\hat{\mathscr{C}}_{\mathfrak{X} ; h}$ is an unbiased estimator for $\mathscr{C}_{\mathscr{X} ; h}$ with $|h|<\mathscr{M}_{M}$, and for $M \rightarrow \infty$ holds

$$
\mathbb{E}\left\|\hat{\mathscr{C}}_{\mathscr{X} ; h}-\mathscr{C}_{\mathscr{X} ; h}\right\|_{\mathcal{S}_{\mathcal{U}^{m}}}^{2}=\mathrm{O}\left((1+|h|) m^{2} p M^{-1}\right) \text {. }
$$

If the first moment $m_{\boldsymbol{X}}$ of $\boldsymbol{X}=\left(X_{k}\right)_{k}$ is unknown and thus also $m_{\mathscr{X}}=\left(m_{\boldsymbol{X}}, \ldots, m_{\boldsymbol{X}}\right)^{T} \in \mathcal{U}^{m}$, we estimate $\mathscr{C}_{\mathscr{X} ; h}$ with $|h|<\mathscr{M}_{M}-1$ by

$$
\hat{\mathscr{C}}_{\mathscr{X} ; h}^{\prime}:= \begin{cases}\frac{1}{\mathscr{M}_{M, h}-1} \sum_{k=m+|h|}^{\tilde{\mathscr{M}}_{M}}\left(\mathscr{X}_{k}-\hat{m}_{\mathscr{X}}\right) \otimes\left(\mathscr{X}_{k+h}-\hat{m}_{\mathscr{X}}^{\prime}\right), & h<0, \\ \frac{1}{\mathscr{M}_{M, h}-1} \sum_{k=m}^{\tilde{\mathscr{M}}_{M, h}}\left(\mathscr{X}_{k}-\hat{m}_{\mathscr{X}}\right) \otimes\left(\mathscr{X}_{k+h}-\hat{m}_{\mathscr{X}}^{\prime}\right), & h \geq 0,\end{cases}
$$

with moment estimators

$$
\hat{m}_{\mathscr{X}}:=\left\{\begin{array}{ll}
\frac{1}{\mathscr{\mathscr { M }}_{M, h}} \sum_{i=m+|h|}^{\tilde{\mathscr{M}}_{M}} \mathscr{X}_{i}, & h<0, \\
\frac{1}{\mathscr{M}_{M, h}} \sum_{i=m}^{\tilde{\mathscr{M}}_{M, h}} \mathscr{X}_{i}, & h \geq 0,
\end{array} \quad \hat{m}_{\mathscr{X}}^{\prime}:= \begin{cases}\frac{1}{\mathscr{M}_{M, h}} \sum_{j=m+|h|}^{\tilde{\mathscr{M}}_{M}} \mathscr{X}_{j+h}, & h<0, \\
\frac{1}{\mathscr{M}_{M, h}} \sum_{j=m}^{\tilde{\mathscr{M}}_{M, h}} \mathscr{X}_{j+h}, & h \geq 0 .\end{cases}\right.
$$


These empirical lag- $h$-covariance operators satisfy also $\hat{\mathscr{C}}_{\mathscr{X} ; h}^{\prime} \in \mathcal{F}_{\mathcal{U}^{m}}$ with $\hat{\mathscr{C}}_{\mathscr{X} ; h}^{\prime *}=\hat{\mathscr{C}}_{\mathscr{X} ;-h}^{\prime}$, and $\hat{\mathscr{C}}_{\mathscr{X}}^{\prime}:=\hat{\mathscr{C}}_{\mathscr{X} ; 0}^{\prime}$ is self-adjoint and positive semi-definite.

Theorem 3.2. Under Assumptions 3.1-3.3 (a), $\hat{\mathscr{C}}_{\mathscr{X} ; h}^{\prime}$ is an unbiased estimator for $\mathscr{C}_{\mathscr{X} ; h}$ with $|h|<\mathscr{M}_{M}-1$ if $\sum_{j, k=1, k \neq j}^{\mathscr{M}_{M, h}} \mathscr{C}_{\mathscr{X} ; j+h-k}=0_{\mathcal{L}_{\mathcal{U}^{m}}}$, and for $M \rightarrow \infty$

$$
\left\|\hat{\mathscr{C}}_{\mathscr{X} ; h}^{\prime}-\mathscr{C}_{\mathscr{X} ; h}\right\|_{\mathcal{S}_{\mathcal{U}} m}^{2}=\mathrm{O}_{\mathbb{P}}\left((1+|h|) m^{2} p M^{-1}\right)
$$

Remarks 3.3. (a) Theorems 3.1-3.2 extend the existing literature regarding the estimation of (lagged) covariance operators in several ways, see e.g. [1], [5], [16], [21] [26], [18], [19], [29], [30]. This is because the upper bounds in both Theorems are derived for lagged covariance operators of processes with arbitrary moments having values in arbitrary separable Hilbert spaces, and since the processes' 'outer dimension' $m$ and simultaneously the lag $h$ is allowed to grow w.r.t. the sample size $M$.

(b) The best achievable rates in Theorems 3.1-3.2 are $\mathrm{O}\left(M^{-1}\right)$ resp. $\mathrm{O}_{\mathbb{P}}\left(M^{-1}\right)$, which apply if $h=$ $h_{M}, m=m_{M}, p=p_{M}$ are bounded or even fixed.

(c) Using $\frac{1}{\mathscr{M}_{M, h}-1}$ instead of $\frac{1}{\mathscr{M}_{M, h}}$ in (3.7) enables to formulate the sufficient condition for unbiasedness in Theorem 3.2. For $h=0$, this condition holds if $\mathscr{C}_{\boldsymbol{X} ; h}=0_{\mathcal{L}_{\mathcal{U}}}$ for all $h \neq 0$ which is due to (2.4) particularly the case if $\boldsymbol{X}$ is a process of i.i.d. random variables.

(d) In Theorem 3.2, we considered convergence in probability instead of convergence in mean since in the proof emerge reciprocals of eigenvalues.

Now, we exemplarily illustrate some upper bounds in Theorem 3.1.

Example 3.1. (a) For $h=m=p=1,(3.6)$ is $\mathrm{O}\left(M^{-1}\right)$, see Remarks 3.3 (b).

(b) For $h=0$, and $m=m_{M}:=\left\lfloor M^{1 / 4}\right\rfloor, p=p_{M}:=\left\lfloor M^{1 / 8}\right\rfloor$ for all $M \in \mathbb{N},(3.6)$ is $\mathrm{O}\left(M^{-3 / 8}\right)$.

(c) Let $h=h_{M}=m=m_{M}=p=p_{M}:=\left\lfloor M^{1 / 8}\right\rfloor$ for all $M \in \mathbb{N}$. Consequently, Assumption 3.3 (a) is satisfied, and (3.6) equals $\mathrm{O}\left(M^{-1 / 2}\right)$.

\subsection{Estimation of lag- $h$-cross-covariance operators}

Herein, we transfer the estimation procedure for lag- $h$-covariance to lag- $h$-cross-covariance operators $\mathscr{C}_{\mathscr{X}, \mathscr{Y} ; h}$. If the processes $\boldsymbol{X}=\left(X_{k}\right)_{k}$ and $\boldsymbol{Y}=\left(Y_{k}\right)_{k}$ in Assumption 3.1 are centered and subsequently also $\mathscr{X}=\left(\mathscr{X}_{k}\right)_{k}$ and $\mathscr{Y}=\left(\mathscr{Y}_{k}\right)_{k}$, we estimate $\mathscr{C}_{\mathscr{X}, \mathscr{Y} ; h}$ with $n-\tilde{\mathscr{M}}_{M} \leq h \leq \tilde{\mathscr{N}}_{N}-m$ by

$$
\hat{\mathscr{C}}_{\mathscr{X}, \mathscr{Y} ; h}:=\frac{1}{\mathscr{L}_{M, N, h}} \sum_{k=\tilde{l}_{m, n, h}}^{\tilde{\mathscr{L}}_{M, N, h}} \mathscr{X}_{k} \otimes \mathscr{Y}_{k+h},
$$

where $\tilde{l}_{m, n, h}:=\max (m, n-h), \tilde{\mathscr{L}}_{M, N, h}:=\min \left(\tilde{\mathscr{M}}_{M}, \tilde{\mathscr{N}}_{N}-h\right)$ and where $\mathscr{L}_{M, N, h}:=\tilde{\mathscr{L}}_{M, N, h}+1-\tilde{l}_{m, n, h}$. Thereby, $\hat{\mathscr{C}}_{\mathscr{X}, \mathscr{Y} ; h} \in \mathcal{F}_{\mathcal{U}^{m}, \mathcal{V}^{n}}$ and $\hat{\mathscr{C}}_{\mathscr{X}, \mathscr{Y} ; h}^{*}=\hat{\mathscr{C}}_{\mathscr{Y}, \mathscr{X} ;-h}$. When estimating the lag- $h$-cross-covariance operators, we also impose the following.

Assumption 3.4. The sequences in Assumptions 3.1-3.3 satisfy $\tilde{l}_{m, n, h}=\mathrm{o}\left(\tilde{\mathscr{L}}_{M, N, h}\right)$.

Theorem 3.3. Let Assumptions 3.1-3.4 hold and let $\boldsymbol{X}, \boldsymbol{Y}$ be centered. Then, $\hat{\mathscr{C}}_{\mathscr{X}, \mathscr{Y} ; h}$ is an unbiased estimator for $\mathscr{C}_{\mathscr{X}, \mathscr{Y} ; h}$ with $n-\tilde{\mathscr{M}}_{M} \leq h \leq \tilde{\mathscr{N}}_{N}-m$, and for $h=h_{L}$ with $L=L_{M, N}:=\min (M, N), m=$ $m_{M}, n=n_{N}$ holds for $M, N \rightarrow \infty$ :

$$
\mathbb{E}\left\|\hat{\mathscr{C}}_{\mathscr{X}, \mathscr{Y} ; h}-\mathscr{C}_{\mathscr{X}, \mathscr{Y} ; h}\right\|_{\mathcal{S}_{\mathcal{U}^{m}, \mathcal{V}^{n}}}^{2}=\mathrm{O}\left((1+|h|) m n \mathscr{L}_{M, N, h}^{-1}\right)
$$


If the first moments $m_{\boldsymbol{X}}$ and/or $m_{\boldsymbol{Y}}$ of $\boldsymbol{X}, \boldsymbol{Y}$ in Assumption 3.1 are unknown, thus also $m_{\mathscr{X}}=$ $\left(m_{\boldsymbol{X}}, \ldots, m_{\boldsymbol{X}}\right)^{T} \in \mathcal{U}^{m}$ and/or $m_{\mathscr{Y}}=\left(m_{\boldsymbol{Y}}, \ldots, m_{\boldsymbol{Y}}\right)^{T} \in \mathcal{V}^{n}, \mathscr{C}_{\mathscr{X}, \mathscr{Y} ; h}$ with $n-\tilde{\mathscr{M}}_{M} \leq h \leq \tilde{\mathscr{N}}_{N}-m$ is estimated by

$$
\hat{\mathscr{C}}_{\mathscr{X}, \mathscr{Y} ; h}^{\prime}:=\frac{1}{\mathscr{L}_{M, N, h}-1} \sum_{k=\tilde{l}_{m, n, h}}^{\tilde{\mathscr{L}}_{M, N, h}}\left(\mathscr{X}_{k}-\hat{m}_{\mathscr{X}}\right) \otimes\left(\mathscr{Y}_{k+h}-\hat{m}_{\mathscr{Y}}^{\prime}\right)
$$

if $\tilde{\mathscr{L}}_{M, N, h}>\tilde{l}_{m, n, h}$, with moment estimators

$$
\hat{m}_{\mathscr{X}}:=\frac{1}{\mathscr{L}_{M, N, h}} \sum_{i=\tilde{l}_{m, n, h}}^{\tilde{\mathscr{L}}_{M, N, h}} \mathscr{X}_{i}, \quad \hat{m}_{\mathscr{Y}}^{\prime}:=\frac{1}{\mathscr{L}_{M, N, h}} \sum_{j=\tilde{l}_{m, n, h}}^{\tilde{\mathscr{L}}_{M, N, h}} \mathscr{Y}_{j+h} .
$$

Thereby, $\hat{\mathscr{C}}_{\mathscr{X}, \mathscr{Y} ; h}^{\prime} \in \mathcal{F}_{\mathcal{U}^{m}, \mathcal{V}^{n}}$ and $\hat{\mathscr{C}}_{\mathscr{X}, \mathscr{Y} ; h}^{\prime *}=\hat{\mathscr{C}}_{\mathscr{Y}, \mathscr{X} ;-h}^{\prime}$ for all $h$.

Theorem 3.4. Under Assumptions 3.1-3.4, $\hat{\mathscr{C}}_{\mathscr{X}, \mathscr{Y} ; h}$ is an unbiased estimator for $\mathscr{C}_{\mathscr{X}, \mathscr{Y} ; h}$ with $n-\tilde{\mathscr{M}}_{M} \leq$ $h \leq \tilde{\mathscr{N}}_{N}-m$, and for $h=h_{L}$ with $L=L_{M, N}:=\min (M, N), m=m_{M}$ and $n=n_{N}$ holds for $M, N \rightarrow \infty$ :

$$
\left\|\hat{\mathscr{C}}_{\mathscr{X}, \mathscr{Y} ; h}^{\prime}-\mathscr{C}_{\mathscr{X}, \mathscr{Y} ; h}\right\|_{\mathcal{S}_{\mathcal{U}, \mathcal{V} n}}^{2}=\mathrm{O}_{\mathbb{P}}\left((1+|h|) m n \mathscr{L}_{M, N, h}^{-1}\right)
$$

Remarks 3.4. (a) Although estimating (lagged) cross-covariance operators is widely discussed, see e.g. [36], [5], [2], [18], Theorems 3.3-3.4 are new in many ways. First, both processes can attain values in arbitrary separable Hilbert spaces which do not necessarily need to match, nor do the drawn sample sizes $M, N$. Further, the upper bounds are, as in Theorems 3.1-3.2 for the lagged covariance operators, derived for centered and for not necessarily centered processes, the lag $h$ is allowed to be both fixed and varying w.r.t. the sample sizes, as are the Cartesian powers $m, n$.

(b) The reason that the initial and final value of the sum of our empirical lag- $h$-cross-covariance operators in (3.9) and (3.11) are relatively complicated, is, because $\mathscr{X}_{k}$ and $\mathscr{Y}_{k+h}$ simultaneously have to be well-defined.

(c) Assumption 3.4 ensures that the upper bounds (3.10) and (3.13) are zero sequences if the sequences in Assumptions 3.1-3.3 are chosen appropriately.

(d) The best possible rates in Theorems 3.3-3.4, $\mathrm{O}\left(M^{-1}\right)$ resp. $\mathrm{O}_{\mathbb{P}}\left(M^{-1}\right)$, hold if $h=h_{L}, m=m_{M}, n=$ $n_{N}, p=p_{M}, q=q_{N}$ are bounded, and if $M \asymp N$.

(e) By following the lines in the proof of Theorem 3.4, it becomes clear that omitting the estimation of $\mathscr{X}_{k}$ resp. $\mathscr{Y}_{k+h}$ in (3.12) if $\boldsymbol{X}$ is centered and $m_{\boldsymbol{Y}}$ is unknown resp. if $m_{\boldsymbol{X}}$ is unknown and $\boldsymbol{Y}$ is centered, has no positive effect on the convergence rate (3.13) in Theorem 3.4.

(f) The condition for unbiasedness in Theorem 3.4 is for instance for $h=0$ satisfied if $\boldsymbol{X}, \boldsymbol{Y}$ are independent, subsequently also $\mathscr{X}, \mathscr{Y}$.

As in Example 3.1, we state several possible upper bounds in Theorem 3.3. Such examples can also be applied to Theorem 3.4 .

Example 3.2. (a) Let $h=m=n=p=q=1$ and $N=N_{M}:=\left\lfloor M^{1 / 2}\right\rfloor$ for all $M$. Then, with $L=\min (M, N),(3.10)$ becomes $\mathrm{O}\left(L^{-1}\right)=\mathrm{O}\left(M^{-1 / 2}\right)$.

(b) Let $h_{k}:=\left\lfloor k^{1 / 8}\right\rfloor, m_{k}:=\left\lfloor k^{1 / 8}\right\rfloor, n_{k}:=\left\lfloor k^{1 / 6}\right\rfloor, p_{k}:=\left\lfloor k^{1 / 10}\right\rfloor, q_{k}:=\left\lfloor k^{1 / 9}\right\rfloor$ for all $k$, and $N=N_{M}:=$ $\left\lfloor M^{1 / 2}\right\rfloor$ for all $M$. Then, $h=h_{L} \sim M^{1 / 16}, m=m_{M} \sim M^{1 / 8}, n=n_{N} \sim M^{1 / 12}, p=p_{M} \sim M^{1 / 10}, q=$ $q_{N} \sim M^{1 / 18}$, hence, $\tilde{l}_{m, n, h} \sim \max \left(M^{1 / 8}, M^{1 / 12}-M^{1 / 16}\right) \sim M^{1 / 8}$ and $\tilde{\mathscr{L}}_{M, N, h} \sim \min \left(M^{9 / 10}+M^{1 / 8}\right.$, $\left.M^{4 / 9}+M^{1 / 8}-M^{1 / 16}\right) \sim M^{4 / 9}$. Consequently, all Assumptions in Theorem 3.3 hold, and (3.10) is $\mathrm{O}\left(M^{1 / 16+1 / 8+1 / 12-4 / 9}\right)=\mathrm{O}\left(M^{-25 / 144}\right)$. 


\subsection{Estimation of principle components}

Herein, we examine the estimation procedure of the principle components of lag-0-covariance operators $\mathscr{C}_{\mathscr{X}}=\mathscr{C}_{\mathscr{X} ; 0}$ of the $\mathcal{U}^{m}$-valued processes $\mathscr{X}=\left(\mathscr{X}_{k}\right)_{k \in \mathbb{Z}}$ in Assumption 3.1 (a). Thereby, $\left(\mathfrak{c}_{j}\right)_{j \in \mathbb{N}},\left(\hat{\mathfrak{c}}_{j}\right)_{j \in \mathbb{N}}$ resp. $\left(\hat{\mathfrak{c}}_{j}^{\prime}\right)_{j \in \mathbb{N}}$ are the eigenfunction and $\left(c_{j}\right)_{j \in \mathbb{N}},\left(\hat{c}_{j}\right)_{j \in \mathbb{N}}$ resp. $\left(\hat{c}_{j}^{\prime}\right)_{j \in \mathbb{N}}$ the associated w.l.o.g. monotonically decreasing eigenvalue sequences of $\mathscr{C}_{\mathscr{X}}, \hat{\mathscr{C}}_{\mathscr{X}}=\hat{\mathscr{C}}_{\mathscr{X}} ; 0$ in (3.5) resp. $\hat{\mathscr{C}}_{\mathscr{X}}^{\prime}=\hat{\mathscr{C}}_{\mathscr{X} ; 0}^{\prime}$ in (3.7). Also, since the vector lengths $m$ of the elements of $\mathscr{X}=\left(\mathscr{X}_{k}\right)_{k}$ can vary w.r.t. $M$, we occasionally write $c_{j}=c_{j, m}$ and $\mathfrak{c}_{j}=\mathfrak{c}_{j, m}$.

At first, due to [5], Lemma 4.2, for any $j \in \mathbb{N}$ holds

$$
\left|\hat{c}_{j}-c_{j}\right| \leq\left\|\hat{\mathscr{C}}_{\mathscr{X}}-\mathscr{C}_{\mathscr{X}}\right\|_{\mathcal{L}_{\mathcal{U}} m}, \quad\left|\hat{c}_{j}^{\prime}-c_{j}\right| \leq\left\|\hat{\mathscr{C}}_{\mathscr{X}}^{\prime}-\mathscr{C}_{\mathscr{X}}\right\|_{\mathcal{L}_{\mathcal{U}^{m}}} .
$$

Corollary 3.1. Let Assumptions 3.1-3.2 (a) hold. Then,

$$
\sup _{j \in \mathbb{N}}\left(\hat{c}_{j}^{\prime}-c_{j}\right)^{2}=\mathrm{O}_{\mathbb{P}}\left(m^{2} p M^{-1}\right),
$$

and if $\boldsymbol{X}=\left(X_{k}\right)_{k}$ in Assumption 3.1 (a) is centered,

$$
\mathbb{E}\left(\sup _{j \in \mathbb{N}}\left(\hat{c}_{j}-c_{j}\right)^{2}\right)=\mathrm{O}\left(m^{2} p M^{-1}\right) .
$$

We proceed with estimating the eigenfunctions $\mathfrak{c}_{j}$ of $\mathscr{C}_{\mathscr{C}}$ by $\hat{\mathfrak{c}}_{j}$ if $\mathscr{X}$ is centered and by $\hat{\mathfrak{c}}_{j}^{\prime}$ if the first moment of $\mathscr{X}$ is unknown. Eigenfunctions are unambiguously determined except for their sign, why it is reasonable to estimate $\mathfrak{c}_{j}$ by

$$
\breve{\mathfrak{c}}_{j}:=\operatorname{sgn}\left\langle\hat{\mathfrak{c}}_{j}, \mathfrak{c}_{j}\right\rangle_{\mathcal{U}^{m}} \hat{\mathfrak{c}}_{j} \quad \text { resp. } \quad \breve{\mathfrak{c}}_{j}^{\prime}:=\operatorname{sgn}\left\langle\hat{\mathfrak{c}}_{j}^{\prime}, \mathfrak{c}_{j}\right\rangle_{\mathcal{U}^{m}} \hat{\mathfrak{c}}_{j}^{\prime}
$$

where 'sgn' is the sign function.

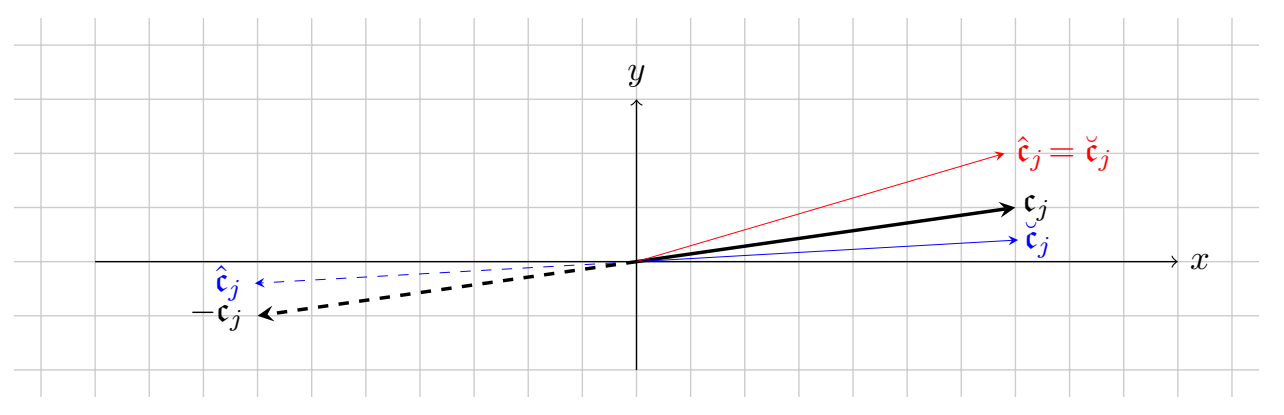

Figure 4: Estimation of $\mathfrak{c}_{j}$ by $\breve{\mathfrak{c}}_{j}$, exemplified in $\mathbb{R}^{2}$

However, using $\breve{\mathfrak{c}}_{j}$ resp. $\breve{\mathfrak{c}}_{j}^{\prime}$ as estimator for $\mathfrak{c}_{j}$ is problematic, since $\breve{\mathfrak{c}}_{j} \not \perp \mathfrak{c}_{j}$ a.s. resp. $\breve{\mathfrak{c}}_{j}^{\prime} \not \perp \mathfrak{c}_{j}$ a.s., thus $\operatorname{sgn}\left\langle\hat{\mathfrak{c}}_{j}, \mathfrak{c}_{j}\right\rangle_{\mathcal{U}^{m}} \neq 0$ a.s. and $\operatorname{sgn}\left\langle\hat{\mathfrak{c}}_{j}^{\prime}, \mathfrak{c}_{j}\right\rangle_{\mathcal{U}^{m}} \neq 0$ a.s. is not guaranteed for fixed $j, M$, so if $\breve{\mathfrak{c}}_{j} \perp \mathfrak{c}_{j}$ resp. $\breve{\mathfrak{c}}_{j}^{\prime} \perp \mathfrak{c}_{j}$, one is not able to allocate a unique estimator for $\mathfrak{c}_{j}$. This feature, though, was inevitable in various conversions leading to asymptotic upper bounds of the estimation errors for operators of $L^{2}[0,1]$-valued (G)ARCH and linear, invertible processes in [29] resp. [30].

We bypass this problem by modifying $\breve{\mathfrak{c}}_{j}$ and $\breve{\mathfrak{c}}_{j}^{\prime}$ as follows. Let $\left(u_{i}\right)_{i \in \mathbb{N}}$ be a CONS of $\mathcal{U}^{m}$ and let $\left(\zeta_{i}\right)_{i \in \mathbb{N}}$ be a sequence of i.i.d., $\mathcal{N}(0,1)$-distributed random variables, independent of the observations of $\boldsymbol{X}$. Then, for all $j, M$,

$$
\breve{\mathfrak{c}}_{j}^{\dagger}:=\hat{\mathfrak{c}}_{j}+\sum_{i=1}^{\infty} \frac{\zeta_{i} u_{i}}{i^{2} M} \quad \text { and } \quad{\breve{\mathfrak{c}_{j}^{\prime}}}_{j}^{\dagger}:=\hat{\mathfrak{c}}_{j}^{\prime}+\sum_{i=1}^{\infty} \frac{\zeta_{i} u_{i}}{i^{2} M}
$$


are well-defined with $\breve{\mathfrak{c}}_{j}^{\dagger} \not \mathcal{\prime} \mathfrak{c}_{j}$ a.s. resp. $\breve{\mathfrak{c}}_{j}^{\prime \dagger} \not \perp \mathfrak{c}_{j}$ a.s., thus $\operatorname{sgn}\left\langle\breve{\mathfrak{c}}_{j}^{\dagger}, \mathfrak{c}_{j}\right\rangle_{\mathcal{U}^{m}} \neq 0$ a.s. and $\operatorname{sgn}\left\langle\breve{c}_{j}^{\prime \dagger}, \mathfrak{c}_{j}\right\rangle_{\mathcal{U}^{m}} \neq 0$ a.s. Hence, we estimate $\mathfrak{c}_{j}$ by

$$
\begin{aligned}
\breve{\mathfrak{c}}_{j}^{\ddagger} & :=\left[\mathbf{1}_{\mathbb{R} \backslash\{0\}}\left(\operatorname{sgn}\left\langle\breve{\mathfrak{c}}_{j}^{\dagger}, \mathfrak{c}_{j}\right\rangle_{\mathcal{U}^{m}}\right) \operatorname{sgn}\left\langle\breve{\mathfrak{c}}_{j}^{\dagger}, \mathfrak{c}_{j}\right\rangle_{\mathcal{U}^{m}}+\mathbf{1}_{\{0\}}\left(\operatorname{sgn}\left\langle\breve{\mathfrak{c}}_{j}^{\dagger}, \mathfrak{c}_{j}\right\rangle_{\mathcal{U}^{m}}\right)\right] \hat{\mathfrak{c}}_{j}, \\
\breve{\mathfrak{c}}_{j}^{\prime \ddagger} & :=\left[\mathbf{1}_{\mathbb{R} \backslash\{0\}}\left(\operatorname{sgn}\left\langle\breve{\mathfrak{c}}_{j}^{\prime \dagger}, \mathfrak{c}_{j}\right\rangle_{\mathcal{U}^{m}}\right) \operatorname{sgn}\left\langle\breve{\mathfrak{c}}_{j}^{\prime \dagger}, \mathfrak{c}_{j}\right\rangle_{\mathcal{U}^{m}}+\mathbf{1}_{\{0\}}\left(\operatorname{sgn}\left\langle\breve{\mathfrak{c}}_{j}^{\dagger \dagger}, \mathfrak{c}_{j}\right\rangle_{\mathcal{U}^{m}}\right)\right] \hat{\mathfrak{c}}_{j}^{\prime},
\end{aligned}
$$

where $\mathbf{1}_{A}(\cdot)$ stands for the indicator function of a set $A$. These estimators satisfy

$$
\breve{\mathfrak{c}}_{j}^{\ddagger}=\operatorname{sgn}\left\langle\breve{\mathfrak{c}}_{j}^{\dagger}, \mathfrak{c}_{j}\right\rangle_{\mathcal{U}^{m}} \hat{\mathfrak{c}}_{j} \text { a.s. } \quad \text { resp. } \quad \breve{\mathfrak{c}}_{j}^{\prime \ddagger}=\operatorname{sgn}\left\langle\breve{\mathfrak{c}}_{j}^{\prime \dagger}, \mathfrak{c}_{j}\right\rangle_{\mathcal{U}^{m}} \hat{\mathfrak{c}}_{j}^{\prime} \text { a.s. }
$$

For stating upper bounds of estimation errors when using the estimators in (3.16), following technical preliminaries are needed. Due to [5], Lemma 4.3,

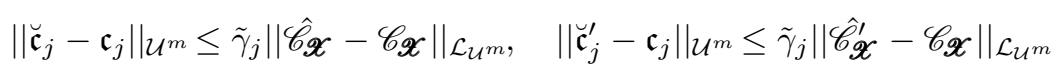

for any $j \in \mathbb{N}$ if the eigenspace of $c_{j}$ is one-dimensional, where $\tilde{\gamma}_{1}:=2 \sqrt{2} \gamma_{1}, \tilde{\gamma}_{j}:=2 \sqrt{2} \max \left(\gamma_{j-1}, \gamma_{j}\right)$ for $j>1$, and where

$$
\gamma_{j}:=\left(c_{j}-c_{j+1}\right)^{-1}, \quad j \in \mathbb{N}
$$

Assumption 3.5. $\mathscr{C}_{\mathscr{X}}$ is injective, and the eigenvalues of $\mathscr{C}_{\mathscr{X}}$ satisfy $c_{j} \neq c_{j+1}$ and $\kappa(j)=c_{j}$ for all $j \in \mathbb{N}$ where $\kappa: \mathbb{R} \rightarrow \mathbb{R}$ is a convex function.

Under Assumption 3.5 holds both

$$
c_{1}>c_{2}>\cdots>0
$$

and for any sequence $\left(k_{j}\right)_{j} \subseteq \mathbb{N}$ with $k=k_{M}=\Omega(1)$,

$$
\sup _{j \leq k} \tilde{\gamma}_{j}=\gamma_{k}
$$

Lemma 3.2. Let Assumptions 3.1-3.2 (a) and 3.5 hold. Further, let $\left(k_{j}\right)_{j} \subseteq \mathbb{N}$ be a sequence with $k=k_{M}=\Omega(1)$, and let $\gamma_{j, m}:=1 /\left(c_{j, m}-c_{j+1, m}\right)$. Then,

$$
\begin{aligned}
\left\|\breve{\mathfrak{c}}_{j}^{\prime}-\mathfrak{c}_{j}\right\|_{\mathcal{U}^{m}}^{2} & =\mathrm{O}_{\mathbb{P}}\left(\gamma_{j, m}^{2} m^{2} p M^{-1}\right), \quad \forall j \in \mathbb{N}, \\
\sup _{j \leq k}\left\|\breve{\mathfrak{c}}_{j}^{\prime}-\mathfrak{c}_{j}\right\|_{\mathcal{U}^{m}}^{2} & =\mathrm{O}_{\mathbb{P}}\left(\gamma_{k, m}^{2} m^{2} p M^{-1}\right),
\end{aligned}
$$

and if $\boldsymbol{X}=\left(X_{k}\right)_{k}$ in Assumption 3.1 (a) is centered,

$$
\begin{aligned}
\mathbb{E}\left\|\breve{\mathfrak{c}}_{j}-\mathfrak{c}_{j}\right\|_{\mathcal{U}^{m}}^{2} & =\mathrm{O}\left(\gamma_{j, m}^{2} m^{2} p M^{-1}\right), \quad \forall j \in \mathbb{N}, \\
\mathbb{E}\left(\sup _{j \leq k}\left\|\breve{\mathfrak{c}}_{j}-\mathfrak{c}_{j}\right\|_{\mathcal{U}^{m}}^{2}\right) & =\mathrm{O}\left(\gamma_{k, m}^{2} m^{2} p M^{-1}\right) .
\end{aligned}
$$

These statements for the 'classical' eigenfunction estimators (3.15) can be transferred to our advanced estimators (3.17), (3.18).

Theorem 3.5. Let Assumptions 3.1-3.2 (a) and 3.5 hold, and let $\left(k_{j}\right)_{j} \subseteq \mathbb{N}$ be a sequence with $k=$ $k_{M}=\Omega(1)$. Then,

$$
\begin{aligned}
\left\|\breve{\mathfrak{c}}_{j}^{\prime \ddagger}-\mathfrak{c}_{j}\right\|_{\mathcal{U}^{m}}^{2} & =\mathrm{O}_{\mathbb{P}}\left(\gamma_{j, m}^{2} m^{2} p M^{-1}\right), \quad \forall j \in \mathbb{N} ; \\
\sup _{j \leq k}\left\|\breve{\mathfrak{c}}_{j}^{\ddagger}-\mathfrak{c}_{j}\right\|_{\mathcal{U}^{m}}^{2} & =\mathrm{O}_{\mathbb{P}}\left(\gamma_{k, m}^{2} m^{2} p M^{-1}\right) .
\end{aligned}
$$

Moreover, if $\boldsymbol{X}=\left(X_{k}\right)_{k}$ in Assumption 3.1 (a) is centered,

$$
\begin{aligned}
\mathbb{E}\left\|\breve{\mathfrak{c}}_{j}^{\ddagger}-\mathfrak{c}_{j}\right\|_{\mathcal{U}^{m}}^{2} & =\mathrm{O}\left(\gamma_{j, m}^{2} m^{2} p M^{-1}\right), \quad \forall j \in \mathbb{N} ; \\
\mathbb{E}\left(\sup _{j \leq k}\left\|\breve{\mathfrak{c}}_{j}^{\ddagger}-\mathfrak{c}_{j}\right\|_{\mathcal{U}^{m}}^{2}\right) & =\mathrm{O}\left(\gamma_{k, m}^{2} m^{2} p M^{-1}\right) .
\end{aligned}
$$


Remarks 3.5. (a) Theorem 3.5 and also Lemma 3.2 can be seen as generalizations of results in [5], [21], [26], [28] dealing with estimating eigenfunctions of centered $L^{2}[0,1]$-valued processes.

(b) If $m=m_{M}$ is bounded, the sequences of reciprocal spectral gaps $\left(\gamma_{j, m}\right)_{M}$ are bounded for any $j$. Consequently, (3.28) equals $\mathrm{O}_{\mathbb{P}}\left(M^{-1}\right)$, and $(3.30)$ is $\mathrm{O}\left(M^{-1}\right)$. Moreover, $\left(\gamma_{k, m}\right)_{M}$ is guaranteed to be bounded if $k=k_{M}$ and $m=m_{M}$ are bounded. Then, (3.29) is $\mathrm{O}_{\mathbb{P}}\left(M^{-1}\right)$, and (3.31) is $\mathrm{O}\left(M^{-1}\right)$.

\section{A simulation study}

Herein, we simulate realizations and estimators of lagged covariance and cross-covariance operators of specific processes. To avoid unnecessary complexity, and to ensure vividness of the derived results, we discuss centered processes whose underlying processes attain values in $\mathcal{H}:=L^{2}[0,1]$. In our calculations with the program language $\mathrm{R}$, any $x \in \mathcal{H}$ is evaluated at $t=0, \frac{1}{250}, \ldots, \frac{249}{250}$, and $\langle x, y\rangle_{\mathcal{H}}$ with $x, y \in \mathcal{H}$ is approximated by the Riemann sum $\frac{1}{250} \sum_{t=1}^{250} x\left(\frac{t-1}{250}\right) y\left(\frac{t-1}{250}\right)$.

\subsection{Setup}

For some $m, n \in \mathbb{N}$, let $\mathscr{X}:=\left(\mathscr{X}_{k}\right)_{k \in \mathbb{Z}}$ and $\mathscr{Y}:=\left(\mathscr{Y}_{k}\right)_{k \in \mathbb{Z}}$ be processes with

$$
\mathscr{X}_{k}:=\left(X_{k}, \ldots, X_{k-m+1}\right)^{T} \quad \text { resp. } \quad \mathscr{Y}_{k}:=\left(Y_{k}, \ldots, Y_{k-n+1}\right)^{T}, \quad \forall k \in \mathbb{Z},
$$

where $\boldsymbol{X}:=\left(X_{k}\right)_{k \in \mathbb{Z}}$ and $\boldsymbol{Y}:=\left(Y_{k}\right)_{k \in \mathbb{Z}}$ are processes which satisfy a.s.

$$
\begin{gathered}
X_{k}=\alpha\left(X_{k-1}\right)+\varepsilon_{k}, \quad \forall k \in \mathbb{Z}, \\
Y_{k}=\beta\left(X_{k}\right)+\varepsilon_{k}, \quad \forall k \in \mathbb{Z} .
\end{gathered}
$$

Thereby, $\varepsilon_{k}$ are defined as in Example 2.1 for any $k \in \mathbb{Z}$, and $\alpha, \beta: \mathcal{H} \rightarrow \mathcal{H}$ are assumed to be integral operators with kernels

$$
a(s, t):=k_{\varepsilon}(s, t) \quad \operatorname{resp} . \quad b(s, t):=2 k_{\varepsilon}(s, t), \quad \forall s, t \in[0,1]
$$

where $k_{\varepsilon ; 0}=k_{\varepsilon}$ is the integral kernel of $\mathscr{C}_{\varepsilon ; 0}=\mathscr{C}_{\varepsilon}$ in $(2.10)$. Since the kernel $a(s, t)$ is bounded, we obtain after $\|\alpha\|_{\mathcal{S}_{\mathcal{H}}}^{2}=\int_{0}^{1} \int_{0}^{1} a^{2}(s, t) \mathrm{d} s \mathrm{~d} t$ and its definition,

$$
\|\alpha\|_{\mathcal{S}_{\mathcal{H}}}=\sqrt{\frac{3}{2}-\ln (2)} .
$$

Also, $\|\alpha\|_{\mathcal{L}_{\mathcal{H}}} \leq\|\alpha\|_{\mathcal{S}_{\mathcal{H}}}<1$ implies that (4.2) has the unique stationary solution

$$
X_{k}=\sum_{j=0}^{\infty} \alpha^{j}\left(\varepsilon_{k-j}\right), \quad \forall k \in \mathbb{Z}
$$

where $\alpha^{0}:=\mathbb{I}_{\mathcal{H}}$ is the identity operator, and where the series converges in $L_{\mathcal{H}}^{4}$ and a.s., see [5]. Thus $\left(X_{k}\right)_{k}$ is a stationary, centered $L_{\mathcal{H}}^{4}$-valued $A R(1)$ process, and it can be shown it is even $L_{\mathcal{H}}^{4}$ - $m$-approximable, see Lemma 2.1 in [30] for functional $(\mathrm{G}) \mathrm{ARCH}$ processes. Further, due to $(4.3),\left(Y_{k}\right)_{k}$ is a stationary, centered $L_{\mathcal{H}}^{4}$-m-approximable process as well.

After [5], for our AR(1) process $\boldsymbol{X}$ holds $\mathscr{C}_{\boldsymbol{X} ; 0}=\mathscr{C}_{\boldsymbol{X}}=\sum_{j=0}^{\infty} \alpha^{j} \mathscr{C}_{\boldsymbol{\varepsilon}} \alpha^{* j}$ with $\mathscr{C}_{\boldsymbol{\varepsilon} ; 0}=\mathscr{C}_{\boldsymbol{\varepsilon}}$, and $\mathscr{C}_{\boldsymbol{X} ; h}=$ $\alpha^{h} \mathscr{C}_{\boldsymbol{X}}$ for $h \in \mathbb{N}_{0}$. Further, $\mathscr{C}_{\boldsymbol{X} ; h}^{*}=\mathscr{C}_{\boldsymbol{X} ;-h}$ for $h \in \mathbb{Z}$, since $\alpha=\mathscr{C}_{\boldsymbol{\varepsilon}}$ is selfadjoint and commutes with $\mathscr{C}_{\boldsymbol{\varepsilon}}$ and due to the series representation of $\mathscr{C}_{\boldsymbol{X}}$ also with $\mathscr{C}_{\boldsymbol{X}}$, and $\|\alpha\|_{\mathcal{S}_{\mathcal{H}}}<1$ lead to the Neumann series

$$
\mathscr{C}_{\boldsymbol{X} ; h}=\alpha^{|h|+1} \sum_{j=0}^{\infty} \alpha^{2 j}=\alpha^{|h|+1}\left(\mathbb{I}_{\mathcal{H}}-\alpha^{2}\right)^{-1}, \quad \forall h \in \mathbb{Z} .
$$


Moreover, (4.2), (4.3), Lemma 2.2, and (4.7) yield

$$
\mathscr{C}_{\boldsymbol{X}, \boldsymbol{Y} ; h}=\beta \mathscr{C}_{\boldsymbol{X} ; h}, \quad \forall h \in \mathbb{Z} .
$$

For the lag-h-covariance operators $\mathscr{C}_{\mathscr{X} ; h}=\mathbb{E}\left\langle\mathscr{X}_{0}, \boldsymbol{x}\right\rangle_{\mathcal{H}^{m}} \mathscr{X}_{h}$ and the lag- $h$-cross-covariance operators $\mathscr{C}_{\mathscr{X}, \mathscr{Y} ; h}=\mathbb{E}\left\langle\mathscr{X}_{0}, \boldsymbol{x}\right\rangle_{\mathcal{H}^{m}} \mathscr{Y}_{h}$ holds for any $h \in \mathbb{Z}$ and $\boldsymbol{x}:=\left(x_{1}, \ldots, x_{m}\right)^{T} \in \mathcal{H}^{m}$,

$$
\begin{aligned}
\mathscr{C}_{\mathscr{X} ; h}(\boldsymbol{x}) & =\left(\sum_{i=1}^{m} \mathscr{C}_{\boldsymbol{X} ; h+i-1}\left(x_{i}\right), \ldots, \sum_{i=1}^{m} \mathscr{C}_{\boldsymbol{X} ; h+i-m}\left(x_{i}\right)\right)^{T} \in \mathcal{H}^{m} \\
\mathscr{C}_{\mathscr{X}, \mathscr{Y} ; h}(\boldsymbol{x}) & =\left(\sum_{i=1}^{m} \mathscr{C}_{\boldsymbol{X}, \boldsymbol{Y} ; h+i-1}\left(x_{i}\right), \ldots, \sum_{i=1}^{m} \mathscr{C}_{\boldsymbol{X}, \boldsymbol{Y} ; h+i-n}\left(x_{i}\right)\right)^{T} \in \mathcal{H}^{n} .
\end{aligned}
$$

Remarks 4.1. For extensive works dealing with functional AR(MA) processes, we refer to [5], [43] and also [16], [1], [7], [8], [34], [18] from a technical point of view, and [10], [25], [40] for methods combined with applications.

\subsection{Simulation of realizations of our processes}

Hereinafter, we simulate realizations of $\left(\mathscr{X}_{k}\right)_{k}$ and $\left(\mathscr{Y}_{k}\right)_{k}$ in (4.1). To do this, we firstly simulate innovations in (2.9).
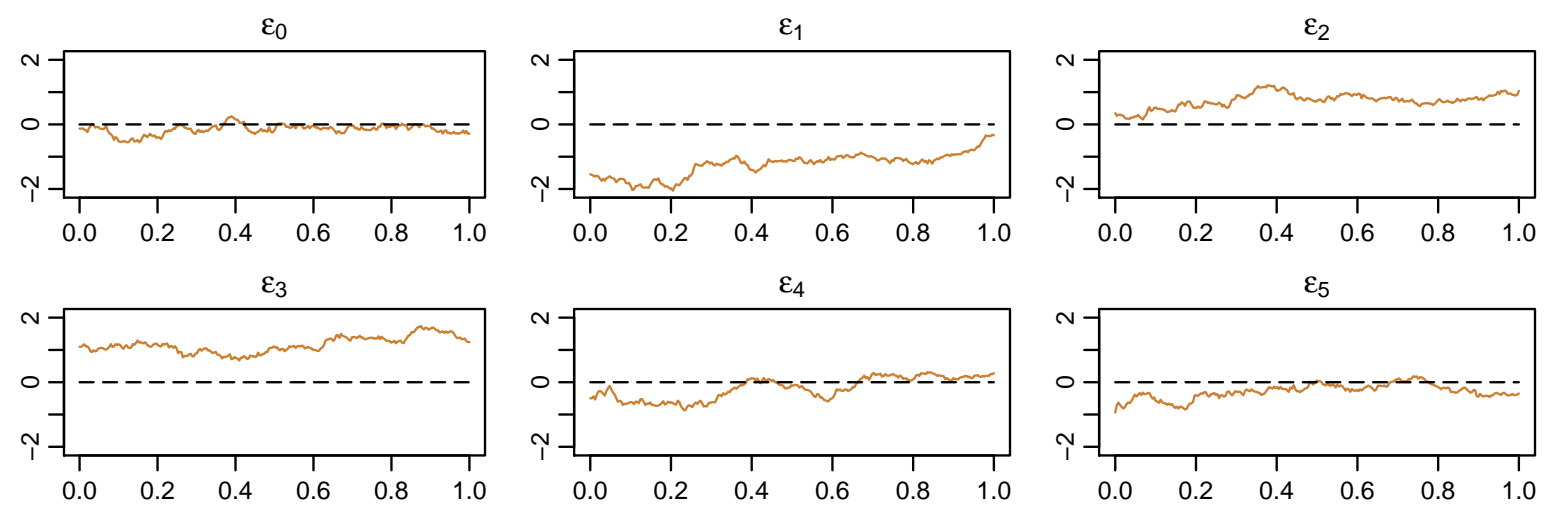

Figure 5: Realizations of the innovations $\varepsilon_{0}, \ldots, \varepsilon_{5}$ in $(2.9)$.

These simulated realizations then can be plugged into the equations (4.2) and (4.3) of the underlying $\operatorname{AR}(1)$ process $\left(X_{k}\right)_{k}$ of $\left(\mathscr{X}_{k}\right)_{k}$ and the derived underlying process $\left(Y_{k}\right)_{k}$ of $\left(\mathscr{Y}_{k}\right)_{k}$. But before we do so, an initial value of $X_{0}$ has to be simulated which can be approximated sufficiently well as follows.

Lemma 4.1. Let $A \in \mathcal{L}_{\mathcal{H}}$ with $\|A\|_{\mathcal{L}_{\mathcal{H}}}<1$, let $\left(\varepsilon_{k}\right)_{k \in \mathbb{Z}}$ be an i.i.d., centered $L_{\mathcal{H}}^{\nu}$-process for $\nu>0$, and let $Z_{k}=A\left(Z_{k-1}\right)+\varepsilon_{k}$ and $\tilde{Z}_{k}=A\left(\tilde{Z}_{k-1}\right)+\varepsilon_{k}$ for any $k \in \mathbb{Z}$ hold, where $\tilde{Z}_{0} \in \mathcal{H}$ is a deterministic value. Then, for some $\rho \in(0,1)$,

$$
\mathbb{E}\left\|Z_{N}-\tilde{Z}_{N}\right\|_{\mathcal{H}}^{\nu}=\mathrm{O}\left(\rho^{N}\right)
$$

Remarks 4.2. Lemma 4.1 can be shown for functional AR(MA) processes with arbitrary order(s) in any separable Hilbert space, see [30], Corollary 4.1 for functional $(\mathrm{G}) \mathrm{ARCH}$ processes. 

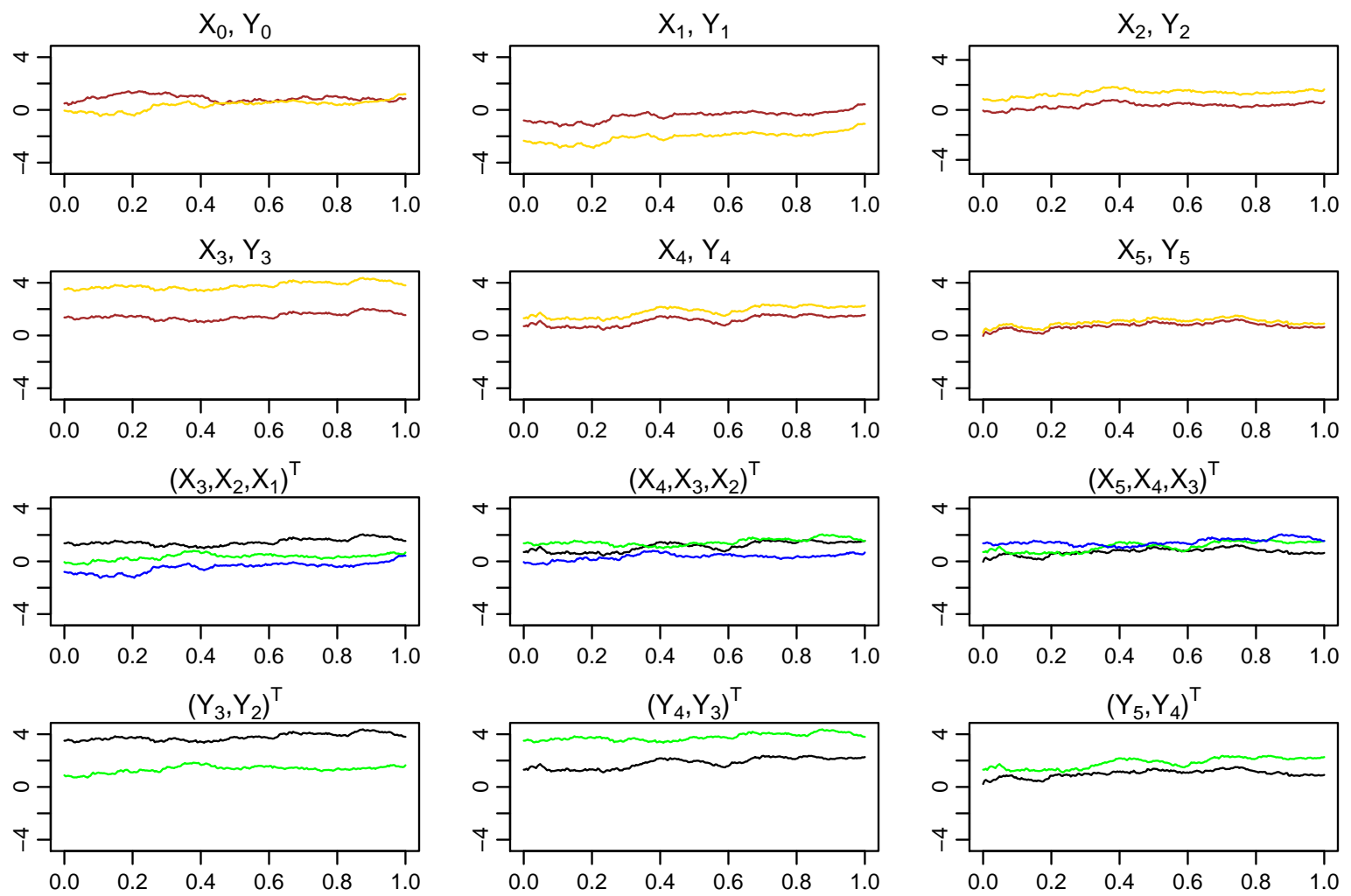

Figure 6: Six consecutive realizations of $\left(X_{k}\right)_{k}$ (bordeaux) and $\left(Y_{k}\right)_{k}$ (gold) in the first two rows. $X_{0}$ was approximated by $\tilde{Z}_{100}$ in Lemma 4.1 with $A=\alpha, \varepsilon_{k}$ for $k=1, \ldots, 100$ as in $(2.9)$ and $\tilde{Z}_{0}:=0_{\mathcal{H}}$, and $X_{1}, \ldots, X_{5}$ and $Y_{0}, \ldots, Y_{5}$ were obtained by applying (4.2) resp. (4.3) with the innovations in Fig 5 . Then, $X_{0}, \ldots, X_{5}$ and $Y_{0}, \ldots, Y_{5}$ were plugged into the equations in (4.1) with $m=3$ and $n=2$, leading to three consecutive realizations of $\left(\mathscr{X}_{k}\right)_{k}=\left(\left(X_{k}, X_{k-1}, X_{k-2}\right)^{T}\right)_{k}$ (third row) and of $\left(\mathscr{Y}_{k}\right)_{k}=\left(\left(Y_{k}, Y_{k-1}\right)^{T}\right)_{k}$ (fourth row). The first resp. the second components of both the realizations of $\left(\mathscr{X}_{k}\right)_{k}$ and $\left(\mathscr{Y}_{k}\right)_{k}$ are highlighted in black resp. green, and the third component of $\left(\mathscr{X}_{k}\right)_{k}$ in blue.

\subsection{Simulation of our operators}

In this section, we illustrate certain lag- $h$-covariance operators $\mathscr{C}_{\mathscr{X}} ; h$ and lag- $h$-cross-covariance operators $\mathscr{C}_{\mathscr{X}, \mathscr{Y} ; h}$ of the centered processes $\mathscr{X}=\left(\mathscr{X}_{k}\right)_{k}$ and $\mathscr{Y}=\left(\mathscr{Y}_{k}\right)_{k}$ in Section 4.2 with Cartesian powers $m=3$ resp. $n=2$, and also simulate estimators for these operators for fixed as well as increasing $h, m, n$.

Due to the infinite series (4.7) consisting of operators, precisely calculating the lag- $h$-covariance and lag- $h$-cross-covariance operators $\mathscr{C}_{\boldsymbol{X} ; h}$ resp. $\mathscr{C}_{\boldsymbol{X}, \boldsymbol{Y} ; h}$ of the processes $\boldsymbol{X}=\left(X_{k}\right)_{k}$ and $\boldsymbol{Y}=\left(Y_{k}\right)_{k}$ is impossible. However, $\mathscr{C}_{\boldsymbol{X} ; h}$ and $\mathscr{C}_{\boldsymbol{X}, \boldsymbol{Y} ; h}$ can for sufficiently large $K \in \mathbb{N}$ and any $h \in \mathbb{Z}$ be well approximated by

$$
\tilde{\mathscr{C}}_{\boldsymbol{X} ; h ; K}:=\alpha^{|h|+1} \sum_{j=0}^{K} \alpha^{2 j} \quad \operatorname{resp} . \quad \tilde{\mathscr{C}}_{\boldsymbol{X}, \boldsymbol{Y} ; h ; K}:=\beta \alpha^{|h|+1} \sum_{j=0}^{K} \alpha^{2 j} .
$$

This is due to the fact that submultiplicity of $\|\cdot\| \mathcal{S}_{\mathcal{H}},\|\alpha\|_{\mathcal{S}_{\mathcal{H}}}<1$ and the formulas of the geometric sum and series lead with $c:=\left(1-\|\alpha\|_{\mathcal{S}_{\mathcal{H}}}^{2}\right)^{-1}$ for any $h, K$ to

$$
\left\|\tilde{\mathscr{C}}_{\mathbf{X} ; h ; K}-\mathscr{C}_{\boldsymbol{X} ; h}\right\|_{\mathcal{S}_{\mathcal{H}}}<c\|\mid \alpha\|_{\mathcal{S}_{\mathcal{H}}}^{2 K+3} \quad \text { and } \quad\left\|\tilde{\mathscr{C}}_{\boldsymbol{X}, \boldsymbol{Y} ; h ; K}-\mathscr{C}_{\boldsymbol{X}, \boldsymbol{Y} ; h}\right\|_{\mathcal{S}_{\mathcal{H}}}<2 c\|\alpha\|_{\mathcal{S}_{\mathcal{H}}}^{2 K+4}
$$

where $\beta=2 \alpha$ after (4.4) was used in the second inequality. Also, the components of $\mathscr{C}_{\mathscr{X} ; h}$ and $\mathscr{C}_{\mathscr{X}, \mathscr{Y} ; h}$ cannot be expressed independently of any argument $\boldsymbol{x}:=\left(x_{1}, \ldots, x_{m}\right)^{T} \in \mathcal{H}^{m}$, except when all of the argument's components match. With $\left(A_{1}(\boldsymbol{x}), \ldots, A_{m}(\boldsymbol{x})\right):=\left(A_{1}, \ldots, A_{m}\right)(\boldsymbol{x})$ for operators $A_{1}, \ldots, A_{m}$ with 
domain $\mathcal{H}^{m}, \mathscr{C}_{\boldsymbol{X} ; h}=\mathscr{C}_{\boldsymbol{X} ;-h}$ and $\mathscr{C}_{\boldsymbol{X}, \boldsymbol{Y} ; h}=\mathscr{C}_{\boldsymbol{X}, \boldsymbol{Y} ;-h}$ for any $h$, for, e.g., $\mathscr{C}_{\mathscr{X} ; 0}$ and $\mathscr{C}_{\mathscr{X}, \mathscr{Y} ;-1}$ with $m=3, n=2$ holds for any $\boldsymbol{x}=(x, x, x) \in \mathcal{H}^{3}$ due to $(4.9),(4.10)$,

$$
\begin{gathered}
\mathscr{C}_{\mathscr{X} ; 0}(\boldsymbol{x})=\left(\left(\mathscr{C}_{\boldsymbol{X} ; 0}+\mathscr{C}_{\boldsymbol{X} ; 1}+\mathscr{C}_{\boldsymbol{X} ; 2}, \mathscr{C}_{\boldsymbol{X} ; 0}+2 \mathscr{C}_{\boldsymbol{X} ; 1}, \mathscr{C}_{\boldsymbol{X} ; 0}+\mathscr{C}_{\boldsymbol{X} ; 1}+\mathscr{C}_{\boldsymbol{X} ; 2}\right)(x)\right)^{T} \\
\mathscr{C}_{\mathscr{X}, \mathscr{Y} ;-1}(\boldsymbol{x})=\left(\left(\mathscr{C}_{\boldsymbol{X}, \boldsymbol{Y} ; 0}+2 \mathscr{C}_{\boldsymbol{X}, \boldsymbol{Y} ; 1}, \mathscr{C}_{\boldsymbol{X}, \boldsymbol{Y} ; 0}+\mathscr{C}_{\boldsymbol{X}, \boldsymbol{Y} ; 1}+\mathscr{C}_{\boldsymbol{X}, \boldsymbol{Y} ; 2}\right)(x)\right)^{T}
\end{gathered}
$$
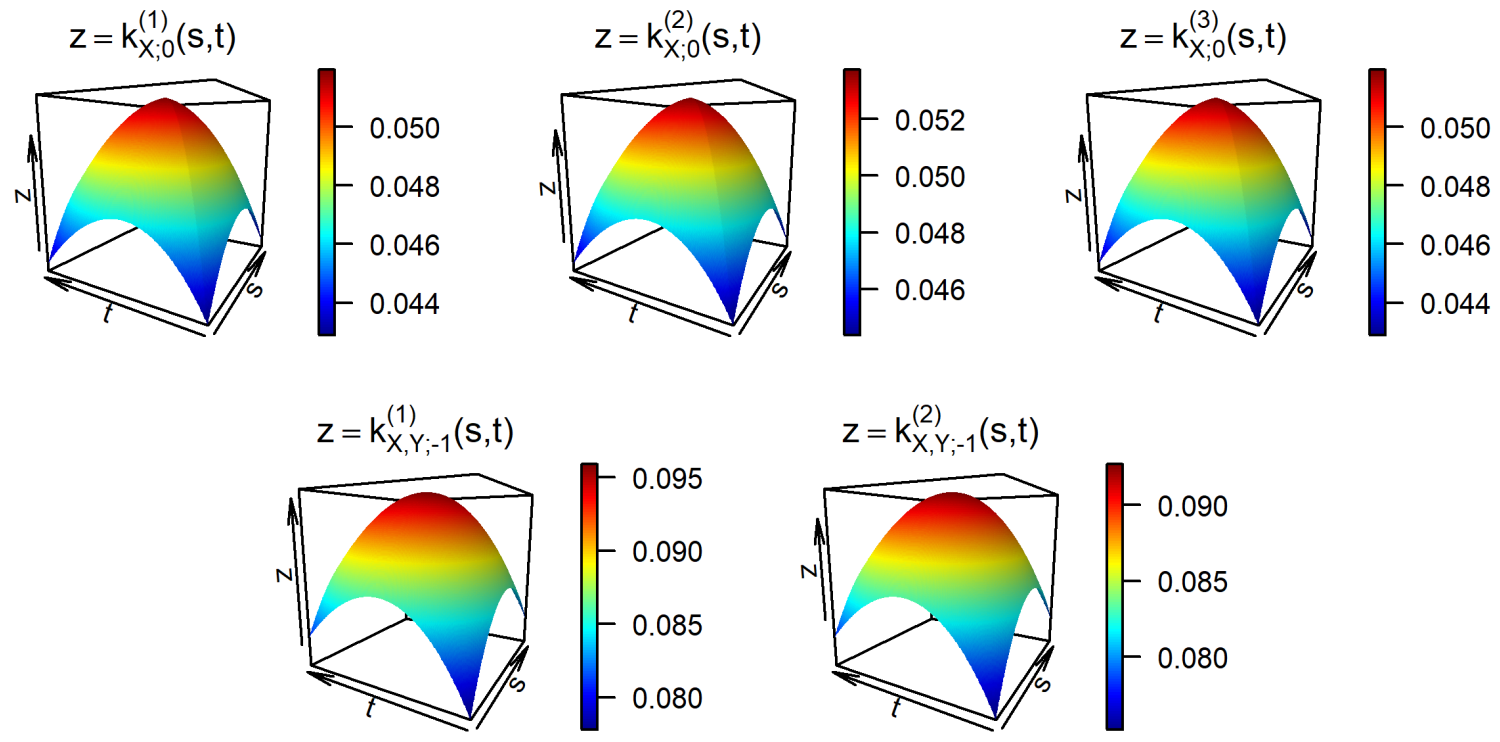

Figure 7: The integral kernels $k_{\mathscr{X} ; 0}^{(1)}, k_{\mathscr{X} ; 0}^{(2)}, k_{\mathscr{X} ; 0}^{(3)}$ (first row) and $k_{\mathscr{X}, \mathscr{Y} ;-1}^{(1)}, k_{\mathscr{X}, \mathscr{Y} ;-1}^{(2)}$ (second row) of the operators in the three resp. two components of $\mathscr{C}_{\mathscr{X} ; 0}$ in (4.13) resp. $\mathscr{C}_{\mathscr{X}, \mathscr{Y} ;-1}$ in (4.14). These kernels result by the associated sum of the integral kernels $k_{\boldsymbol{X} ; 0}, k_{\boldsymbol{X} ; 1}, k_{\boldsymbol{X} ; 2}$ and $k_{\boldsymbol{X}, \boldsymbol{Y} ; 0}, k_{\boldsymbol{X}, \boldsymbol{Y} ; 1}, k_{\boldsymbol{X}, \boldsymbol{Y} ; 2}$ of the operators $\mathscr{C}_{\boldsymbol{X} ; 0}, \mathscr{C}_{\boldsymbol{X} ; 1}, \mathscr{C}_{\boldsymbol{X} ; 2}$ resp. $\mathscr{C}_{\boldsymbol{X}, \boldsymbol{Y} ; 0}, \mathscr{C}_{\boldsymbol{X}, \boldsymbol{Y} ; 1}, \mathscr{C}_{\boldsymbol{X}, \boldsymbol{Y} ; 2}$ which were approximated by their respective operators in (4.12) with $K=100$.

In order to illustrate estimators for the operators in the components of $\mathscr{C}_{\mathscr{X} ; 0}(\boldsymbol{x})$ in $(4.13)$ and $\mathscr{C}_{\mathscr{X}, \mathscr{Y} ;-1}(\boldsymbol{x})$ in (4.14), and to estimate $\mathscr{C}_{\mathscr{X} ; h}$ and $\mathscr{C}_{\mathscr{X}, \mathscr{Y} ; h}$ for fixed and varying $h, m, n$ in general, where $h \geq 0$ is imposed w.l.o.g., we generate $X_{1}, \ldots, X_{M}$ and $Y_{1}, \ldots, Y_{N}$ of the processes $\boldsymbol{X}$ resp. $\boldsymbol{Y}$ in Section 4.1 with $M=N$. This leads to the values $\mathscr{X}_{m}, \ldots, \mathscr{X}_{\tilde{\mathscr{M}}}$ of $\mathscr{X}$ and $\mathscr{Y}_{n}, \ldots, \mathscr{Y}_{\tilde{N}}$ of $\mathscr{Y}$ with $\tilde{\mathscr{M}}=\tilde{\mathscr{M}}_{M}=M$ and $\tilde{\mathscr{N}}=\tilde{\mathscr{N}}_{N}=M$, thus with $\mathscr{M}=\mathscr{M}_{M}=M-m+1$ resp. $\mathscr{N}=\mathscr{N}_{N}=M-n+1$. Due to centeredness of $\boldsymbol{X}$ and $\boldsymbol{Y}$, the operators $\mathscr{C}_{\boldsymbol{X} ; h}$ in (4.13) and $\mathscr{C}_{\boldsymbol{X}, \boldsymbol{Y} ; h}$ in (4.14) with $h=0,1,2$ are estimated by the classical estimators $\hat{\mathscr{C}}_{\boldsymbol{X} ; h}$ resp. by $\hat{\mathscr{C}}_{\boldsymbol{X}, \boldsymbol{Y} ; h}$ with integral kernels

$$
\begin{aligned}
\hat{k}_{\boldsymbol{X} ; h}(s, t) & :=\frac{1}{M-h} \sum_{k=1}^{M-h} X_{k}(s) X_{k+h}(t), \quad \forall s, t \in[0,1], \\
\text { resp. } \quad \hat{k}_{\boldsymbol{X}, \boldsymbol{Y} ; h}(s, t) & :=\frac{1}{M-h} \sum_{k=1}^{M-h} X_{k}(s) Y_{k+h}(t), \quad \forall s, t \in[0,1] .
\end{aligned}
$$



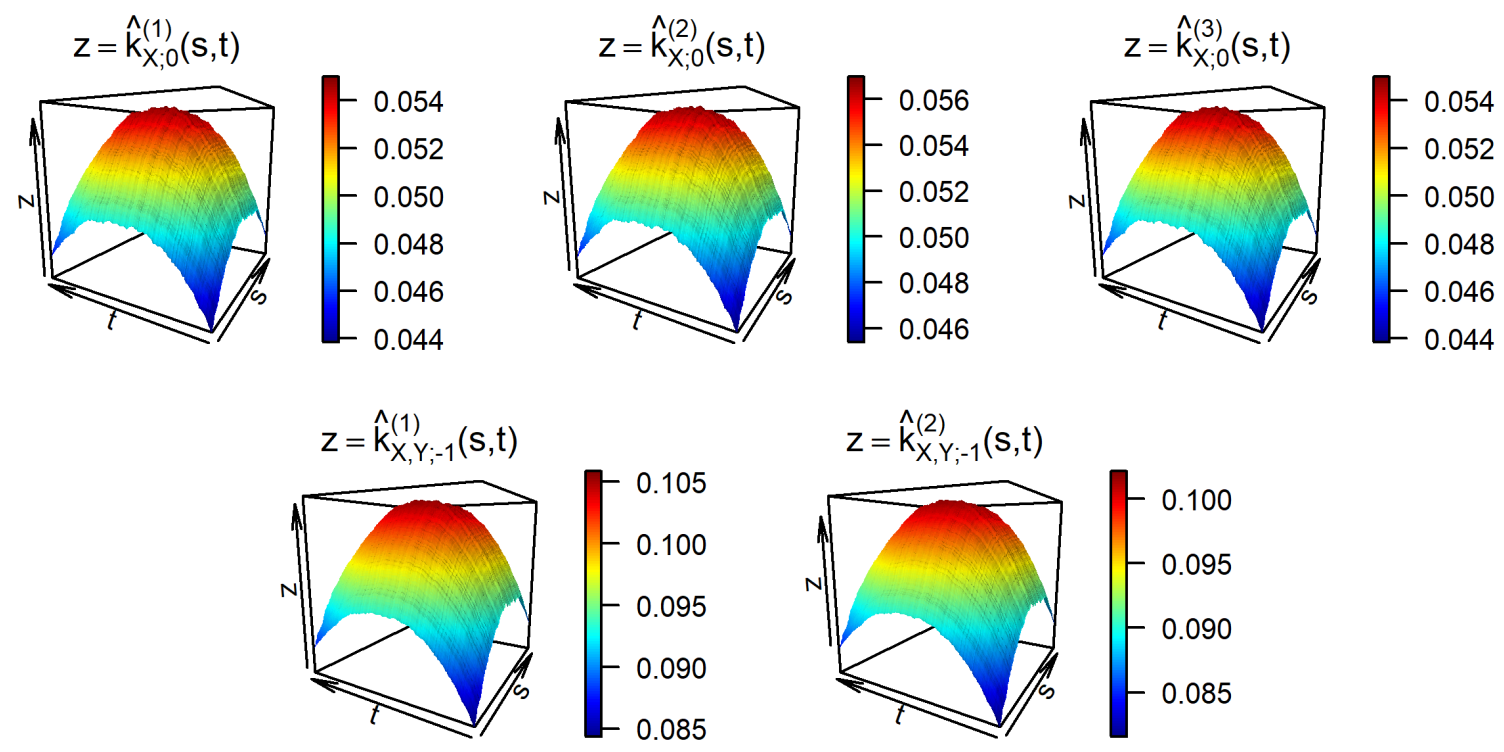

Figure 8: The estimators $\hat{k}_{\mathscr{X} ; 0}^{(1)}, \hat{k}_{\mathscr{X} ; 0}^{(2)}, \hat{k}_{\mathscr{X} ; 0}^{(3)}$ (first row) and $\hat{k}_{\mathscr{X}, \mathscr{Y} ;-1}^{(1)}, \hat{k}_{\mathscr{X}, \mathscr{Y} ;-1}^{(2)}$ (second row) for the integral kernels $k_{\mathscr{X} ; 0}^{(1)}, k_{\mathscr{X} ; 0}^{(2)}, k_{\mathscr{X} ; 0}^{(3)}$ resp. $k_{\mathscr{X}, \mathscr{Y} ;-1}^{(1)}, k_{\mathscr{X}, \mathscr{Y} ;-1}^{(2)}$ of the operators in the three resp. two components of $\mathscr{C}_{\mathscr{X} ; 0}$ in $(4.13)$ resp. $\mathscr{C}_{\mathscr{X}, \mathscr{Y} ;-1}$ in (4.14). These estimators result by the associated sum of the estimators $\hat{k}_{\boldsymbol{X} ; 0}, \hat{k}_{\boldsymbol{X} ; 1}, \hat{k}_{\boldsymbol{X} ; 2}$ in (4.15) and $\hat{k}_{\boldsymbol{X}, \boldsymbol{Y} ; 0}, \hat{k}_{\boldsymbol{X}, \boldsymbol{Y} ; 1}, \hat{k}_{\boldsymbol{X}, \boldsymbol{Y} ; 2}$ in (4.16) with $M=1000$ for the operators $\mathscr{C}_{\boldsymbol{X} ; 0}, \mathscr{C}_{\boldsymbol{X} ; 1}, \mathscr{C}_{\boldsymbol{X} ; 2}$ resp. $\mathscr{C}_{\boldsymbol{X}, \boldsymbol{Y} ; 0}, \mathscr{C}_{\boldsymbol{X}, \boldsymbol{Y} ; 1}, \mathscr{C}_{\boldsymbol{X}, \boldsymbol{Y} ; 2}$.

Finally, in Table 1, we list estimation errors for the operators $\mathscr{C}_{\mathscr{X} ; h}$ and $\mathscr{C}_{\mathscr{X}, \mathscr{Y} ; h}$ of the processes $\mathscr{X}:=\left(\mathscr{X}_{k}\right)_{k \in \mathbb{Z}}$ and $\mathscr{Y}:=\left(\mathscr{Y}_{k}\right)_{k \in \mathbb{Z}}$ defined in (4.1) for several sample sizes $M=N$ and also various $h, m, n$ which may depend on $M$, with $h \geq 0$ w.l.o.g. Due to centeredness of $\mathscr{X}$ and $\mathscr{Y}$, as estimators for $\mathscr{C}_{\mathscr{X} ; h}$ and $\mathscr{C}_{\mathscr{X}, \mathscr{Y} ; h}, \hat{\mathscr{C}}_{\mathscr{X} ; h}$ in (3.5) resp. $\hat{\mathscr{C}}_{\mathscr{X}, \mathscr{Y} ; h}$ in (3.9) are used, which, due to our processes' definition and $h \geq 0$ are represented by

$$
\begin{gathered}
\hat{\mathscr{C}}_{\mathscr{X} ; h}=\frac{1}{M-h-m+1} \sum_{k=m}^{M-h} \mathscr{X}_{k} \otimes \mathscr{X}_{k+h}, \\
\text { resp. } \quad \hat{\mathscr{C}}_{\mathscr{X}, \mathscr{Y} ; h}=\frac{1}{M-h-\max (m, n-h)+1} \sum_{k=\max (m, n-h)}^{M-h} \mathscr{X}_{k} \otimes \mathscr{Y}_{k+h} .
\end{gathered}
$$

In order to calculate the estimation errors, the equations

$$
\begin{aligned}
&\left\|\hat{\mathscr{C}}_{\mathscr{X} ; h}-\mathscr{C}_{\mathscr{X} ; h}\right\|_{\mathcal{S}_{\mathcal{H}^{m}}}^{2}=\sum_{i=1}^{m} \sum_{j=1}^{m}\left\|\hat{\mathscr{C}}_{\boldsymbol{X} ; h+i-j}-\mathscr{C}_{\boldsymbol{X} ; h+i-j}\right\|_{\mathcal{S}_{\mathcal{H}}}^{2}, \\
&\left\|\hat{\mathscr{C}} \mathscr{X}, \mathscr{Y} ; h_{1}-\mathscr{C}_{\mathscr{X}, \mathscr{Y} ; h}\right\|_{\mathcal{S}_{\mathcal{H}^{m}, \mathcal{H}^{n}}}^{2}=\sum_{i=1}^{m} \sum_{j=1}^{n}\left\|\hat{\mathscr{C}}_{\boldsymbol{X}, \boldsymbol{Y} ; h+i-j}-\mathscr{C}_{\boldsymbol{X}, \boldsymbol{Y} ; h+i-j}\right\|_{\mathcal{S}_{\mathcal{H}}}^{2}
\end{aligned}
$$

are utilized, where $\hat{\mathscr{C}}_{\boldsymbol{X} ; h+i-j}$ and $\hat{\mathscr{C}}_{\boldsymbol{X}, \boldsymbol{Y} ; h+i-j}$ equal $\hat{\mathscr{C}}_{\mathscr{X} ; h}$ in (4.17) resp. $\hat{\mathscr{C}}_{\mathscr{X}, \mathscr{Y} ; h}$ in (4.18) with $\mathscr{X}_{k}, \mathscr{X}_{k+h}$ and $\mathscr{Y}_{k+h}$ replaced by $X_{k+1-i}, X_{k+h+1-j}$ resp. $Y_{k+h+1-j}$ for all $i, j$. Thereby, the equations (4.19) and (4.20) follow from the definition of the given norms and operators (see also (4.9), (4.10)). 


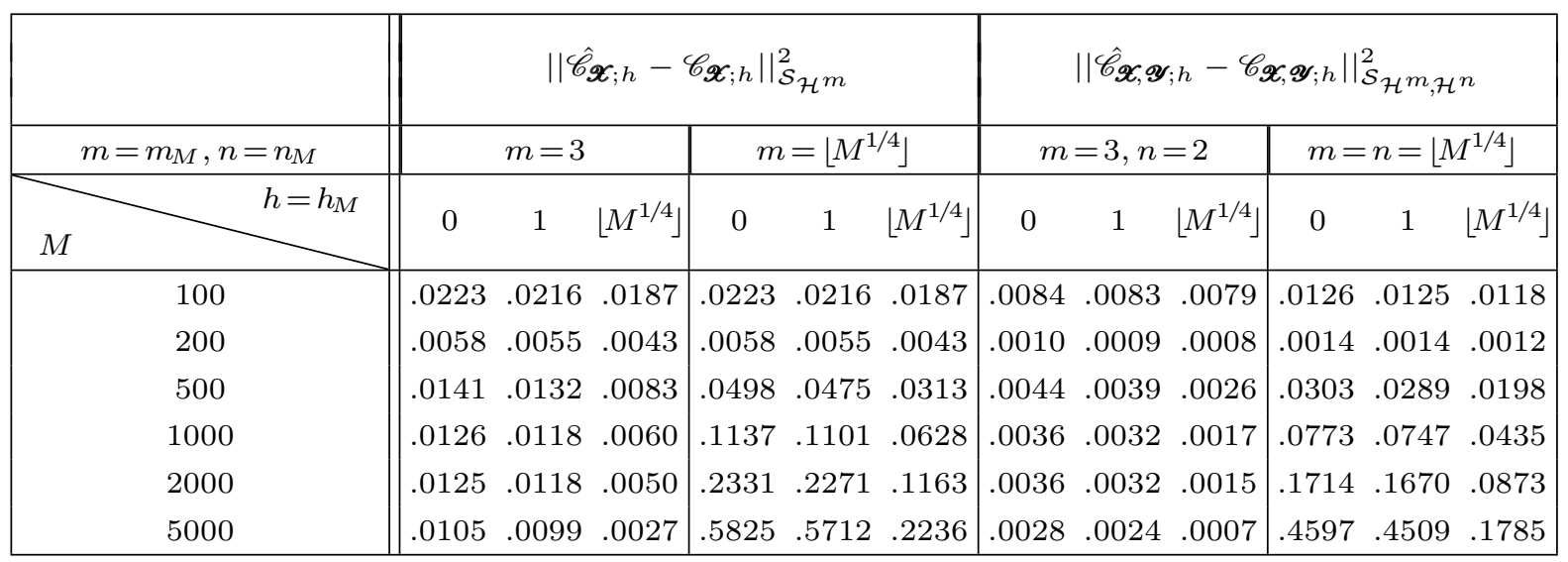

Table 1: Simulation of $\left\|\hat{\mathscr{C}}_{\mathscr{X} ; h}-\mathscr{C}_{\mathscr{X} ; h}\right\|_{\mathcal{S}_{\mathcal{H}} m}^{2}$ in (4.19), $\left\|\hat{\mathscr{C}}_{\mathscr{X}, \mathscr{Y} ; h}-\mathscr{C}_{\mathscr{X}, \mathscr{Y} ; h}\right\|_{\mathcal{S}_{\mathcal{H}}{ }^{m}, \mathcal{H}^{n}}^{2}$ in (4.20) for various sample sizes $M$, lags $h$ and Cartesian powers $m, n$, with $\mathscr{C}_{\boldsymbol{X} ; h+i-j}$ and $\mathscr{C}_{\boldsymbol{X}, \boldsymbol{Y} ; h+i-j}$ approximated by $\tilde{\mathscr{C}}_{\boldsymbol{X} ; h+i-j ; 100}$ resp. $\tilde{\mathscr{C}}_{\boldsymbol{X}, \boldsymbol{Y} ; h+i-j ; 100}$ in $(4.12)$.

Remarks 4.3. All parameters in the simulation study with estimation errors in Table 1 are chosen so that the prerequisites of Theorems 3.1 and 3.3 are satisfied. These errors run for growing sample size $M$ below or as the asymptotic upper bounds in Theorems 3.1 and 3.3. Up to $M=200$, probably due to fortunate random errors, the calculated errors decrease for fixed resp. increase for increasing $m=m_{M}, n=n_{M}$ and any $h=h_{M}$ as expected. That the errors for increasing $m=m_{M}, n=n_{M}$ not yet visibly approach zero could be because $M$ is either too small, or that small values of the estimators or the operators to be estimated are rounded to zero, leading to larger estimation errors. Also, the estimation errors for the lag- $h$-cross-covariance are smaller as for the lag- $h$-covariance operators due to their definiton.

\section{Conclusions}

This article proposes estimators for lagged covariance and cross-covariance operators and the principle components of (lag-0-)covariance operators of processes with values in (Cartesian products of) separable Hilbert spaces where the focus lies on the asymptotic upper bounds of the estimation errors. All estimators are stated for centered processes and for those with an unknown mean. The asymptotic upper bounds allow both the processes' Cartesian powers and the lag to be fixed or to increase w.r.t. the sample size, and the principle components are estimated individually as well as uniformly. Our findings are useful whenever one is concerned about the dependence within one or between two processes having values in (Cartesian products of) Hilbert spaces, or one has to derive asymptotic upper bounds of estimation errors where the given estimators rely on empirical (lagged) covariance or cross-covariance operators, see [2], [30] for latter. These findings can also be applied to covariance and cross-covariance operators of random variables in separable Hilbert spaces, and since $\mathbb{R}^{n}$ endowed with the canonical inner product is a separable Hilbert space for any $n \in \mathbb{N}$, also to conventional (lagged) covariance and cross-covariance matrices.

It would be interesting to deduce our results also on separable Banach spaces, see, e.g., [39] who dealt with the estimation of AR operators in Banach spaces, to derive the asymptotic distribution of our estimation errors (see [36]) as well as their asymptotic lower bounds. 


\section{Proofs}

Proof of Lemma 2.1. See [5], and [22], sections 7.2-7.3.

Proof of Lemma 2.2. Both parts follow from the definition of the lag-h-(cross-)covariance operators and elementary conversions, where the assertion regarding independence in (a) holds due to (2.4).

Proof of Lemma 3.1. $\hat{m}_{\mathscr{X}}$ is an unbiased estimator for $m_{\mathscr{X}}$ due to its definition. $\boldsymbol{X}=\left(X_{k}\right)_{k}$ and $\mathscr{X}=\left(\mathscr{X}_{k}\right)_{k}$ are stationary, and $X_{1+h}, X_{1+h ; h}$ are independent for all $h$. Thus, similar to [20], due to $m_{\mathscr{X}}=\left(m_{\boldsymbol{X}}, \ldots, m_{\boldsymbol{X}}\right)^{T} \in \mathcal{U}^{m},(2.4)$, Cauchy-Schwarz inequality, $\mathscr{Z}_{k}:=\mathscr{X}_{k}-m_{\mathscr{X}}$ and $Z_{k}:=X_{k}-m_{\boldsymbol{X}}$ for any $k$, holds with $\tilde{\mathscr{M}}=\tilde{\mathscr{M}}_{M}, \mathscr{M}=\mathscr{M}_{M}$ in Assumption 3.1:

$$
\begin{aligned}
\mathbb{E}\left\|\hat{m}_{\mathscr{X}}-m_{\mathscr{X}}\right\|_{\mathcal{U}^{m}}^{2} & =\mathscr{M}^{-2} \sum_{i, j=m}^{\tilde{\mathscr{M}}} \mathbb{E}\left\langle\mathscr{Z}_{i}, \mathscr{Z}_{j}\right\rangle_{\mathcal{U}^{m}}=m \mathscr{M}^{-2} \sum_{i, j=1}^{\mathscr{M}} \mathbb{E}\left\langle Z_{i}, Z_{j}\right\rangle_{\mathcal{U}} \\
& =m \mathscr{M}^{-1}\left[\nu_{2, \mathcal{U}}\left(Z_{1}\right)+2 \sum_{h=1}^{\mathscr{M}-1} \frac{\mathscr{M}-h}{\mathscr{M}} \mathbb{E}\left\langle Z_{1}, X_{1+h}-X_{1+h ; h}\right\rangle_{\mathcal{U}}\right] \\
& \leq m \mathscr{M}^{-1} \nu_{2, \mathcal{U}}\left(Z_{1}\right)\left[1+2 \sum_{h=1}^{\infty} \nu_{2, \mathcal{U}}\left(X_{1+h}-X_{1+h ; h}\right)\right] \\
& =\mathrm{O}\left(m \mathscr{M}^{-1}\right)=\mathrm{O}\left(m p M^{-1}\right)
\end{aligned}
$$

where the last two steps hold due to $L_{\mathcal{U}}^{4}$-approximibility of $\left(X_{k}\right)_{k}$ and (3.3).

In various conversions when deriving the upper bounds of the estimation errors for our operators, we use the following two Lemmas.

Lemma 6.1. Let $\left(\mathcal{H},\|\cdot\|_{\mathcal{H}}\right)$ be a separable Hilbert space. Also, let $\left(S_{k}\right)_{k \in \mathbb{Z}}$ be a stationary $L_{\mathcal{H}}^{4}$-process, and for some $l \in \mathbb{N}, \mathscr{S}_{k}:=\left(S_{f(k, 1)}, \ldots, S_{f(k, l)}\right)^{T}$ for all $k$ and some function $f: \mathbb{Z} \times\{1, \ldots, l\} \rightarrow \mathbb{Z}$. Then,

$$
\nu_{4, \mathcal{H}^{l}}\left(\mathscr{S}_{k}\right) \leq \sqrt{l} \nu_{4, \mathcal{H}}\left(S_{j}\right), \quad \forall j, k .
$$

Proof. From the definition of $\mathscr{S}_{k}$ and $\nu_{4, \mathcal{H}^{l}}(\cdot)$, from stationarity of the $L_{\mathcal{H}^{4}}^{4}$-process $\left(S_{k}\right)_{k}$ and CauchySchwarz inequality follows

$$
\nu_{4, \mathcal{H}^{l}}^{4}\left(\mathscr{S}_{k}\right)=\mathbb{E}\left[\left(\sum_{m=1}^{l}\left\|S_{f(k, m)}\right\|_{\mathcal{H}}^{2}\right)^{2}\right] \leq \sum_{m, n=1}^{l} \mathbb{E}\left\|S_{j}\right\|_{\mathcal{H}}^{4}=l^{2} \nu_{4, \mathcal{H}}^{4}\left(S_{j}\right) .
$$

Lemma 6.2. Let Assumption 3.1 hold. Moreover, we define $\mathscr{X}_{m+j ; l}:=\left(X_{m+j p ; l}, \ldots, X_{1+j p ; l}\right)^{T}$ and $\mathscr{Y}_{n+j ; l}:=\left(Y_{n+j q ; l}, \ldots, Y_{1+j q ; l}\right)^{T}$ for any $j, l, m, n, p, q$.

(a) The processes $\left(\mathscr{X}_{k}\right)_{k \in \mathbb{Z}}$ and $\left(\mathscr{Y}_{k}\right)_{k \in \mathbb{Z}}$ satisfy

$$
\sum_{k=1}^{\infty} \nu_{4, \mathcal{U}^{m}}\left(\mathscr{X}_{k}-\mathscr{X}_{k ; k}\right)<\infty \quad \text { resp. } \quad \sum_{k=1}^{\infty} \nu_{4, \mathcal{\nu}^{n}}\left(\mathscr{Y}_{k}-\mathscr{Y}_{k ; k}\right)<\infty .
$$

Thereby, $\left(\mathscr{X}_{k}\right)_{k}$ is $L_{\mathcal{U}^{m}}^{4}$-m-approximable for $p=1$, and $\left(\mathscr{Y}_{k}\right)_{k}$ is $L_{\mathcal{V}^{n}}^{4}$-m-approximable for $q=1$.

(b) For the process $\left(\mathscr{W}_{k, h}\right)_{k \in \mathbb{Z}}$, with $h \in \mathbb{Z}$ and $\mathscr{W}_{k, h}:=\mathscr{X}_{k} \otimes \mathscr{Y}_{k+h}$, holds with $\mathscr{W}_{k, h ; l}:=\mathscr{X}_{k ; l} \otimes \mathscr{Y}_{k+h ; l}$ :

$$
\begin{aligned}
& \sum_{k=1}^{\infty} \nu_{2, \mathcal{S}_{\mathcal{U}}, \mathcal{V} n}\left(\mathscr{W}_{k, h}-\mathscr{W}_{k, h ; k}\right) \\
& \leq \sqrt{m n}\left[\sum_{k=1}^{\infty} \nu_{4, \mathcal{V}}\left(Y_{1}\right) \nu_{4, \mathcal{U}}\left(X_{k}-X_{k ; k}\right)+\nu_{4, \mathcal{U}}\left(X_{1}\right) \nu_{4, \mathcal{V}}\left(Y_{k}-Y_{k ; k}\right)\right] .
\end{aligned}
$$

Moreover, $\left(\mathscr{W}_{k+h}\right)_{k}$ is $L_{\mathcal{U}^{m}, \mathcal{V}^{n}}^{2}$-m-approximable for $h \leq 0$ if $p=q=1$. 
Proof. (a) From the definition of $\mathscr{X}_{k}, \mathscr{X}_{k ; k}, \mathscr{Y}_{k}, \mathscr{Y}_{k ; k}$ for all $k$ follows $\nu_{4, \mathcal{U}^{m}}\left(\mathscr{X}_{k}-\mathscr{X}_{k ; k}\right) \leq \sqrt{m} \nu_{4, \mathcal{U}}\left(X_{k}-\right.$ $\left.X_{k ; k}\right)$ and $\nu_{4, \mathcal{V}^{n}}\left(\mathscr{Y}_{k}-\mathscr{Y}_{k ; k}\right) \leq \sqrt{n} \nu_{4, \mathcal{V}}\left(Y_{k}-Y_{k ; k}\right)$, and thus $(6.2)$. Hence, since $\left(\mathscr{X}_{k}\right)_{k}$ and $\left(\mathscr{Y}_{k}\right)_{k}$ are nonanticipative w.r.t. $\left(\varepsilon_{k}\right)_{k}$ for $p=1$ resp. $q=1,\left(\mathscr{X}_{k}\right)_{k}$ and $\left(\mathscr{Y}_{k}\right)_{k}$ are $L_{\mathcal{U}^{m}}^{4}-m$ - resp. $L_{\mathcal{V}^{n}}^{4}-m$-approximable.

(b) Bilinearity of $\otimes: \mathcal{U}^{m} \times \mathcal{V}^{n} \rightarrow \mathcal{V}^{n}$, Minkowski inequality, $\|\boldsymbol{u} \otimes \boldsymbol{v}\|_{\mathcal{S}_{\mathcal{U}^{m}, \mathcal{V}^{n}}=\|\boldsymbol{u}\| \mathcal{U}^{m}}\|\boldsymbol{v}\|_{\mathcal{V}^{n}}$ for $\boldsymbol{u} \in \mathcal{U}^{m}, \boldsymbol{v} \in$ $\mathcal{V}^{n}$, Cauchy-Schwarz inequality and (6.1) yield

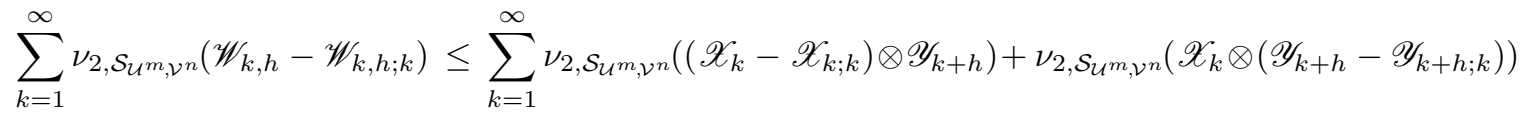

$$
\begin{aligned}
& \leq \sum_{k=1}^{\infty} \nu_{4, \mathcal{U}^{m}}\left(\mathscr{X}_{k}-\mathscr{X}_{k ; k}\right) \nu_{4, \mathcal{\nu}^{n}}\left(\mathscr{Y}_{1}\right)+\nu_{4, \mathcal{U}^{m}}\left(\mathscr{X}_{1}\right) \nu_{4, \mathcal{V}^{n}}\left(\mathscr{Y}_{k}-\mathscr{Y}_{k ; k}\right) \\
& \leq \sqrt{m n}\left[\sum_{k=1}^{\infty} \nu_{4, \mathcal{V}}\left(Y_{1}\right) \nu_{4, \mathcal{U}}\left(X_{k}-X_{k ; k}\right)+\nu_{4, \mathcal{U}}\left(X_{1}\right) \nu_{4, \mathcal{V}}\left(Y_{k}-Y_{k ; k}\right)\right]
\end{aligned}
$$

This is finite due to $L^{4}$-m-approximability of $\left(X_{k}\right)_{k}$ and $\left(Y_{k}\right)_{k}$. Moreover, since $\left(\mathscr{X}_{k}\right)_{k},\left(\mathscr{Y}_{k+h}\right)_{k}$ and thus $\left(\mathscr{W}_{k+h}\right)_{k}$ are non-anticipative w.r.t. $\left(\varepsilon_{k}\right)_{k}$ for $h \leq 0$ if $p=q=1,\left(\mathscr{W}_{k+h}\right)_{k}$ is indeed $L_{\mathcal{S}_{\mathcal{U}}{ }^{\prime}, \mathcal{V}^{n}}^{2}{ }^{-m \text {-approximable }}$ for $h \leq 0$ if $p=q=1$.

Proof of Theorem 3.1. We use ideas from the proof of [21], Theorem 3.1. $\hat{\mathscr{C}}_{\mathscr{X} ; h}$ is an unbiased estimator for $\mathscr{C}_{\mathscr{X} ; h}$ with $|h|<\mathscr{M}=\mathscr{M}_{M}$ due to its definition. Since $\left\|\hat{\mathscr{C}}_{\mathscr{X} ; h}-\mathscr{C}_{\mathscr{X} ; h}\right\|_{\mathcal{S}_{\mathcal{U}} m}=\left\|\hat{\mathscr{C}}_{\mathscr{X} ;-h}-\mathscr{C}_{\mathscr{X} ;-h}\right\|_{\mathcal{S}_{\mathcal{U}} m}$ for all $h$, we show (3.6) for $h \geq 0$ w.l.o.g. Stationarity of $\mathscr{X}$ implies for any $h$ with $0 \leq h<\mathscr{M}_{M}$ where $\mathscr{Z}_{k, h}:=\mathscr{W}_{k, h}-\mathscr{C}_{\mathscr{X} ; h}$ with $\mathscr{W}_{k, h}:=\mathscr{X}_{k} \otimes \mathscr{X}_{k+h}$, and $\mathscr{M}_{M, h}=\mathscr{M}_{M}-|h|:$

$$
\begin{aligned}
\mathbb{E}|| \hat{\mathscr{C}}_{\mathscr{X} ; h}-\mathscr{C}_{\mathscr{X} ; h} \|_{\mathcal{S}_{\mathcal{U}} m}^{2} & =\mathscr{M}_{M, h}^{-2} \sum_{|r|<\mathscr{M}_{M, h}}\left(\mathscr{M}_{M, h}-|r|\right) \mathbb{E}\left\langle\mathscr{Z}_{m, h}, \mathscr{Z}_{m+r, h}\right\rangle_{\mathcal{S}_{\mathcal{U}^{m}}} \\
& \leq 2 \mathscr{M}_{M, h}^{-1} \sum_{r=0}^{\infty} \mathbb{E}\left\langle\mathscr{Z}_{m, h}, \mathscr{Z}_{m+r, h}\right\rangle_{\mathcal{S}_{\mathcal{U}}}
\end{aligned}
$$

Let $\sigma\left(T_{k}, k \in I\right)$ be the $\sigma$-algebra generated by the random variables $T_{k}$ with $k \in I$ where $I \subseteq \mathbb{Z}$ is some index set. From Assumption 3.1 (a), the definition of $\mathscr{X}_{k}$ for any $k$ for some $p \in \mathbb{N}$, and of $\mathscr{W}_{k, h}$ for any $h, k$ follows for $h \geq 0$ :

$$
\mathscr{Z}_{m, h}=\mathscr{W}_{m, h}-\mathscr{C}_{\mathscr{X} ; h} \in \sigma\left(X_{1}, \ldots, X_{m}, X_{1+h p}, \ldots, X_{m+h p}\right) \subseteq \sigma\left(\varepsilon_{m+h p}, \varepsilon_{m+h p-1}, \ldots\right)
$$

where $\left(\varepsilon_{k}\right)_{k}$ is an i.i.d. process, and for any $r \in \mathbb{N}$,

$$
\mathscr{Z}_{m+r, h}=\mathscr{W}_{m+r, h}-\mathscr{C}_{\mathscr{X} ; h} \in \sigma\left(\varepsilon_{m+(h+r) p}, \varepsilon_{m+(h+r) p-1}, \ldots\right) .
$$

Consequently, $\mathscr{Z}_{m, h}$ and $\mathscr{Z}_{m+r, h ; r-h}:=\mathscr{W}_{m+r, h ; r-h}-\mathscr{C}_{\mathscr{X} ; h}$ with $\mathscr{W}_{m+r, h ; r-h}=\mathscr{X}_{m+r ; r-h} \otimes \mathscr{X}_{m+h+r ; r-h}$ (see Lemma 6.2 with $\mathscr{Y}_{k}=\mathscr{X}_{k}, \mathscr{Y}_{k ; l}=\mathscr{X}_{k ; l}$ ) are independent for $r>h$ for any $m, p$. With that being said, and since $\mathscr{Z}_{m, h}$ and $\mathscr{Z}_{m+r, h ; r-h}$ are centered for all $h, k, r$, Cauchy-Schwarz inequality, (2.4) and Lemma 6.2 with $\mathscr{Y}_{k}=\mathscr{X}_{k}, \mathscr{Y}_{k ; l}=\mathscr{X}_{k ; l}$ for all $k, l$, yield for the sum in $(6.4)$ :

$$
\begin{aligned}
& \sum_{r=0}^{\infty} \mathbb{E}\left\langle\mathscr{Z}_{m, h}, \mathscr{Z}_{m+r, h}\right\rangle_{\mathcal{S}_{\mathcal{U}}} \\
& =\sum_{r=0}^{h} \mathbb{E}\left\langle\mathscr{Z}_{m, h}, \mathscr{Z}_{m+r, h}\right\rangle_{\mathcal{S}_{\mathcal{U}^{m}}}+\sum_{r>h} \mathbb{E}\left\langle\mathscr{Z}_{m, h}, \mathscr{W}_{m+r, h}-\mathscr{W}_{m+r, h ; r-h}\right\rangle_{\mathcal{S}_{\mathcal{U}^{m}}} \\
& \leq \nu_{2, \mathcal{S}_{\mathcal{U}^{m}}}\left(\mathscr{Z}_{m, h}\right)\left[(1+h) \nu_{2, \mathcal{S}_{\mathcal{U}} m}\left(\mathscr{Z}_{m, h}\right)+\sum_{k=1}^{\infty} \nu_{2, \mathcal{S}_{\mathcal{U}} m}\left(\mathscr{W}_{k, h}-\mathscr{W}_{k, h ; k}\right)\right]
\end{aligned}
$$




$$
\leq \nu_{2, \mathcal{S}_{\mathcal{U}} m}\left(\mathscr{Z}_{m, h}\right)\left[(1+h) \nu_{2, \mathcal{S}_{\mathcal{U}} m}\left(\mathscr{Z}_{m, h}\right)+2 m \nu_{4, \mathcal{U}}\left(X_{m}\right) \sum_{k=1}^{\infty} \nu_{4, \mathcal{U}}\left(X_{k}-X_{k ; k}\right)\right]
$$

$\nu_{2, \mathcal{S}_{\mathcal{U}} m}^{2}\left(\mathscr{Z}_{m, h}\right):=\mathbb{E}\left\|\mathscr{Z}_{m, h}\right\|_{\mathcal{S}_{\mathcal{U}^{m}}}^{2}=\mathbb{E}\left\|\mathscr{W}_{m, h}\right\|_{\mathcal{S}_{\mathcal{U}^{m}}}^{2}-\left\|\mathscr{C}_{\mathscr{X} ; h}\right\|_{\mathcal{S}_{\mathcal{U}^{m}}}^{2}$ after [22], Theorem 7.2.2, and $\left\|\mathscr{W}_{m, h}\right\|_{\mathcal{S}_{\mathcal{U}^{m}}}^{2}=$ $\left\|\mathscr{X}_{m}\right\|_{\mathcal{U}^{m}}^{2}\left\|\mathscr{X}_{m+h}\right\|_{\mathcal{U}^{m}}^{2}$. Hence, $(2.3),\|\cdot\|_{\mathcal{S}_{\mathcal{U}^{m}}} \leq\|\cdot\|_{\mathcal{N}_{\mathcal{U}} m}$, Cauchy-Schwarz inequality, stationarity of $\left(\mathscr{X}_{k}\right)_{k}$ and (6.1) yield

$$
\nu_{2, \mathcal{S}_{\mathcal{U}^{m}}}^{2}\left(\mathscr{Z}_{m, h}\right) \leq 2 \mathbb{E}\left\|\mathscr{X}_{m}\right\|_{\mathcal{U}^{m}}^{4} \leq 2 m^{2} \nu_{4, \mathcal{U}}^{4}\left(X_{1}\right)
$$

From $(6.4),(6.6),(6.7)$ and $L_{\mathcal{U}}^{4}-m$-approximibility of $\left(X_{k}\right)_{k}$ follows

$$
\mathbb{E}\left\|\hat{\mathscr{C}}_{\mathscr{X} ; h}-\mathscr{C}_{\mathscr{X} ; h}\right\|_{\mathcal{S}_{\mathcal{U}^{m}}}^{2} \leq a(1+h) m^{2} \mathscr{M}_{M, h}^{-1}
$$

for some constant $a$ independent of $h=h_{M}, m=m_{M}, p=p_{M}$ in Assumption 3.2 (a) and thus also of $\mathscr{M}_{M, h}=\mathscr{M}_{M}-|h|$ with $\mathscr{M}_{M, h} \sim p^{-1} M$ after Assumption 3.3 (a) and (3.3). Hence, (3.6) is verified.

Proof of Theorem 3.2. From stationarity of $\mathscr{X}=\left(\mathscr{X}_{k}\right)_{k}$ and bilinearity of $\otimes: \mathcal{U}^{m} \times \mathcal{U}^{m} \rightarrow \mathcal{U}^{m}$ follows for $h$ with $0 \leq h<\mathscr{M}_{M}-1$, and $\mathscr{M}_{M, h}=\mathscr{M}_{M}-|h|$ :

$$
\begin{aligned}
\mathbb{E}\left(\hat{\mathscr{C}}_{\mathscr{X} ; h}^{\prime}\right) & =\frac{1}{\mathscr{M}_{M, h}-1} \sum_{k=1}^{\mathscr{M}_{M, h}} \mathbb{E}\left(\left(\mathscr{X}_{k}-\frac{1}{\mathscr{M}_{M, h}} \sum_{i=1}^{\mathscr{M}_{M, h}} \mathscr{X}_{i}\right) \otimes\left(\mathscr{X}_{k+h}-\frac{1}{\mathscr{M}_{M, h}} \sum_{j=1}^{\mathscr{M}_{M, h}} \mathscr{X}_{j+h}\right)\right) \\
& =\frac{1}{\mathscr{M}_{M, h}\left(\mathscr{M}_{M, h}-1\right)}\left(\mathscr{M}_{M, h}^{2} \mathscr{C}_{\mathscr{X} ; h}-\sum_{i, k=1}^{\mathscr{M}_{M, h}} \mathscr{C}_{\mathscr{X} ; k+h-i}\right) \\
& =\mathscr{C}_{\mathscr{X} ; h}-\frac{1}{\mathscr{M}_{M, h}\left(\mathscr{M}_{M, h}-1\right)} \sum_{\substack{1 \leq i, k \leq \mathscr{M}_{M, h} \\
i \neq k}} \mathscr{C}_{\mathscr{X} ; k+h-i} .
\end{aligned}
$$

Hence, $\hat{\mathscr{C}}_{\mathscr{X} ; h}^{\prime}$ is an unbiased estimator for $\mathscr{C}_{\mathscr{X} ; h}$ for $h$ with $0 \leq h<\mathscr{M}_{M}-1$ if the sum in $(6.8)$ equals $0_{\mathcal{L}_{\mathcal{U}}}$ which can also be shown for $h$ with $1-\mathscr{M}_{M}<h<0$.

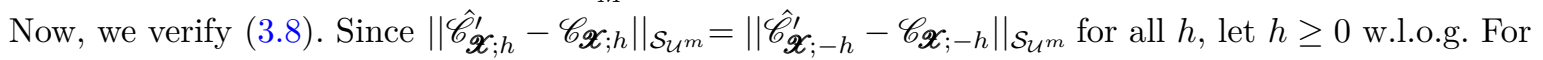
$h<\mathscr{M}_{M}-1$ holds

$$
\begin{aligned}
\hat{\mathscr{C}}_{\mathscr{X} ; h}^{\prime} & =\frac{\mathscr{M}_{M, h}}{\mathscr{M}_{M, h}-1}\left(m_{\mathscr{X}}-\hat{m}_{\mathscr{X}}\right) \otimes\left(m_{\mathscr{X}}-\hat{m}_{\mathscr{X}}^{\prime}\right)+\frac{1}{\mathscr{M}_{M, h}-1} \sum_{j=m}^{\tilde{\mathscr{M}}_{M, h}} \mathscr{U}_{k} \otimes \mathscr{U}_{k+h} \\
& =\frac{\mathscr{M}_{M, h}}{\mathscr{M}_{M, h}-1}\left[\left(m_{\mathscr{X}}-\hat{m}_{\mathscr{X}}\right) \otimes\left(m_{\mathscr{X}}-\hat{m}_{\mathscr{X}}^{\prime}\right)+\hat{\mathscr{C}}_{\mathscr{U} ; h}\right]
\end{aligned}
$$

with $\hat{\mathscr{C}}_{\mathscr{U} ; h}$ as in $(3.5)$ based on a sample $\mathscr{U}_{m}, \ldots, \mathscr{U}_{\tilde{\mathbb{M}}_{M, h}}$ of $\mathscr{U}:=\left(\mathscr{U}_{k}\right)_{k \in \mathbb{Z}}$ where $\mathscr{U}_{k}:=\mathscr{X}_{k}-m_{\mathscr{X}} .(6.9)$, $\mathscr{C}_{\mathscr{X} ; h}=\mathscr{C}_{\mathscr{U} ; h}, \Delta$-inequality, $(a+b+c)^{2} \leq 3\left(a^{2}+b^{2}+c^{2}\right)$ for $a, b, c \in \mathbb{R}$ and $\left\|\boldsymbol{u} \otimes \boldsymbol{u}^{\prime}\right\| \mathcal{S}_{\mathcal{U}^{m}}=\|\boldsymbol{u}\|_{\mathcal{U}^{m}}\left\|\boldsymbol{u}^{\prime}\right\|_{\mathcal{U}^{m}}$ for $\boldsymbol{u}, \boldsymbol{u}^{\prime} \in \mathcal{U}^{m}$ yield

$$
\begin{aligned}
\left\|\hat{\mathscr{C}}_{\mathscr{X} ; h}^{\prime}-\mathscr{C}_{\mathscr{X} ; h}\right\|_{\mathcal{S}_{\mathcal{U}^{m}}}^{2}= & \left\|\frac{1}{\mathscr{M}_{M, h}-1}\left[\mathscr{M}_{M, h}\left(m_{\mathscr{X}}-\hat{m}_{\mathscr{X}}\right) \otimes\left(m_{\mathscr{X}}-\hat{m}_{\mathscr{X}}^{\prime}\right)+\hat{\mathscr{C}}_{\mathscr{U} ; h}-\mathscr{C}_{\mathscr{U} ; h}\right]+\mathscr{C}_{\mathscr{X} ; h}\right\|_{\mathcal{S}_{\mathcal{U}^{m}}}^{2} \\
\leq & \frac{3}{\left(\mathscr{M}_{M, h}-1\right)^{2}}\left[\mathscr{M}_{M, h}^{2}\left\|\hat{m}_{\mathscr{X}}-m_{\mathscr{X}}\right\|_{\mathcal{U}^{m}}^{2}\left\|\hat{m}_{\mathscr{X}}^{\prime}-m_{\mathscr{X}}\right\|_{\mathcal{U}^{m}}^{2}\right. \\
& \left.+\mathscr{M}_{M, h}^{2}\left\|\hat{\mathscr{C}}_{\mathscr{U} ; h}-\mathscr{C}_{\mathscr{U} ; h}\right\|_{\mathcal{S}_{\mathcal{U}^{m}}}^{2}+\left\|\mathscr{C}_{\mathscr{X} ; h}\right\|_{\mathcal{S}_{\mathcal{U}^{m}}}^{2}\right]
\end{aligned}
$$

We have $\left\|\hat{m}_{\mathscr{X}}-m_{\mathscr{X}}\right\|_{\mathcal{U}^{m}}^{2}\left\|\hat{m}_{\mathscr{X}}^{\prime}-m_{\mathscr{X}}\right\|_{\mathcal{U}^{m}}^{2}=\mathrm{O}_{\mathbb{P}}\left(m^{2} \mathscr{M}_{M, h}^{-2}\right)$ after Lemma 3.1, $\left\|\hat{\mathscr{C}}_{\mathscr{U}_{;} h}-\mathscr{C}_{\mathscr{U} ; h}\right\|_{\mathcal{S}_{\mathcal{U}} m}^{2}=\mathrm{O}_{\mathbb{P}}((1+$

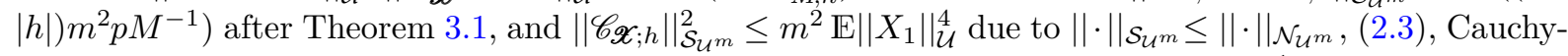
Schwarz inequality and (6.1). Then, under Assumptions 3.1-3.3 (a), thus $\mathscr{M}_{M, h} \sim p^{-1} M$ after (3.3), and

$$
\left\|\hat{\mathscr{C}}_{\mathscr{X} ; h}^{\prime}-\mathscr{C}_{\mathscr{X} ; h}\right\|_{\mathcal{S}_{\mathcal{U}^{m}}}^{2}=\mathrm{O}_{\mathbb{P}}\left(m^{2} \mathscr{M}_{M, h}^{-2}\right)+\mathrm{O}_{\mathbb{P}}\left((1+|h|) m^{2} p M^{-1}\right)+\mathrm{O}\left(m^{2} \mathscr{M}_{M, h}^{-2}\right)
$$




$$
=\mathrm{O}_{\mathbb{P}}\left((1+|h|) m^{2} p M^{-1}\right) .
$$

Proof of Theorem 3.3. $\hat{\mathscr{C}}_{\mathscr{X}, \mathscr{Y} ; h}$ is an unbiased estimator for $\mathscr{C}_{\mathscr{X}, \mathscr{Y} ; h}$ for $h$ with $n-\tilde{\mathscr{M}}_{M} \leq h \leq \tilde{\mathscr{N}}_{N}-m$ by definition. From deliberations in the proof of Theorem 3.1, especially (6.4) and (6.5) with $\mathscr{Z}_{k, h}:=$ $\mathscr{W}_{k, h}-\mathscr{C}_{\mathscr{X}, \mathscr{Y} ; h}$ and $\mathscr{W}_{k, h}:=\mathscr{X}_{k} \otimes \mathscr{Y}_{k+h}$,

$$
\nu_{2, \mathcal{S}_{\mathcal{U}^{m}, \mathcal{V}^{n}}}^{2}\left(\mathscr{Z}_{1, h}\right) \leq 2\left(\mathbb{E}\left\|\mathscr{X}_{m}\right\|_{\mathcal{U}^{m}}^{4} \mathbb{E}\left\|\mathscr{Y}_{n}\right\|_{\mathcal{V}^{n}}^{4}\right)^{1 / 2} \leq 2 m n \nu_{4, \mathcal{U}}^{2}\left(X_{1}\right) \nu_{4, \mathcal{V}}^{2}\left(Y_{1}\right)
$$

similar as in (6.7), and Lemma 6.2 follows

$$
\begin{aligned}
\mathbb{E} \| & \hat{\mathscr{C}}_{\mathscr{X}, \mathscr{Y} ; h}-\mathscr{C}_{\mathscr{X}, \mathscr{Y} ; h} \|_{\mathcal{S}_{\mathcal{U}^{m}, \mathcal{V}^{n}}}^{2} \\
\leq & 2 \mathscr{L}_{M, N, h}^{-1} \nu_{2, \mathcal{S}_{\mathcal{U}^{m}, \mathcal{V}^{n}}}\left(\mathscr{Z}_{1, h}\right)\left[(1+h) \nu_{2, \mathcal{S}_{\mathcal{U}^{m}, \mathcal{V}^{n}}}\left(\mathscr{Z}_{1, h}\right)+\sum_{k=1}^{\infty} \nu_{2, \mathcal{S}_{\mathcal{U}^{m}, \mathcal{V} n}}\left(\mathscr{W}_{k, h}-\mathscr{W}_{k, h ; k}\right)\right] \\
\leq 2 & \sqrt{2} m n \mathscr{L}_{M, N, h}^{-1} \nu_{4, \mathcal{U}}\left(X_{1}\right) \nu_{4, \mathcal{V}}\left(Y_{1}\right) \\
\cdot & {\left[\sqrt{2}(1+h) \nu_{4, \mathcal{U}}\left(X_{1}\right) \nu_{4, \mathcal{V}}\left(Y_{1}\right)+\sum_{k=1}^{\infty} \nu_{4, \mathcal{V}}\left(Y_{1}\right) \nu_{4, \mathcal{U}}\left(X_{k}-X_{k ; k}\right)+\nu_{4, \mathcal{U}}\left(X_{1}\right) \nu_{4, \mathcal{V}}\left(Y_{k}-Y_{k ; k}\right)\right] } \\
\leq & b(1+h) m n \mathscr{L}_{M, N, h}^{-1}
\end{aligned}
$$

for some constant $b$ independent of $M, N$, and thus of all given sequences.

Proof of Theorem 3.4. For $h$ with $n-\tilde{\mathscr{M}}_{M} \leq h \leq \tilde{\mathscr{N}}_{N}-m$ holds

$$
\mathbb{E}\left(\hat{\mathscr{C}}_{\mathscr{X}, \mathscr{Y} ; h}^{\prime}\right)=\hat{\mathscr{C}}_{\mathscr{X}, \mathscr{Y} ; h}-\frac{1}{\mathscr{L}_{M, N, h}\left(\mathscr{L}_{M, N, h}-1\right)} \sum_{\substack{1 \leq i, k \leq \mathscr{L}_{M, N, h} \\ i \neq k}} \mathscr{C}_{\mathscr{X}, \mathscr{Y} ; k+h-i}
$$

similar as in the proof of Theorem 3.2. Thus, $\hat{\mathscr{C}}_{\mathscr{X}, \mathscr{Y} ; h}^{\prime}$ is an unbiased estimator for $\mathscr{C}_{\mathscr{X}, \mathscr{Y} ; h}$ for these $h$ if the sum above is $0_{\mathcal{L}_{\mathcal{U}^{m}, \mathcal{V}^{n}}}$. Moreover, as in Theorem 3.2,

$$
\hat{\mathscr{C}}_{\mathscr{X}, \mathscr{Y} ; h}^{\prime}=\frac{\mathscr{L}_{M, N, h}}{\mathscr{L}_{M, N, h}-1}\left[\left(m_{\mathscr{X}}-\hat{m}_{\mathscr{X}}\right) \otimes\left(m_{\mathscr{Y}}-\hat{m}_{\mathscr{Y}}^{\prime}\right)+\hat{\mathscr{C}}_{\mathscr{U}, \mathscr{V} ; h}\right]
$$

with $\hat{\mathscr{C}}_{\mathscr{U}, \mathscr{Y} ; h}$ defined in (3.9) based on samples $\mathscr{U}_{m}, \ldots, \mathscr{U}_{\tilde{\mathscr{M}}_{M}}$ of $\mathscr{U}:=\left(\mathscr{U}_{k}\right)_{k \in \mathbb{Z}}$ and $\mathscr{V}_{n}, \ldots, \mathscr{V}_{\tilde{N}}$ of $\mathscr{V}:=\left(\mathscr{V}_{k}\right)_{k \in \mathbb{Z}}$ with $\mathscr{U}_{k}:=\mathscr{X}_{k}-m_{\mathscr{X}}$ resp. $\mathscr{V}_{k}:=\mathscr{Y}_{k}-m_{\mathscr{Y}}$. Arguments in the proofs of Theorem 3.2-3.3 imply together with the assertions of Lemma 3.1, Theorem 3.3, (2.3), (6.10) and $\mathscr{C}_{\mathscr{U}, \mathscr{Y} ; h}=\mathscr{C}_{\mathscr{X}, \mathscr{Y} ; h}$ as claimed:

$$
\begin{aligned}
\left\|\hat{\mathscr{C}}_{\mathscr{X}, \mathscr{Y} ; h}^{\prime}-\mathscr{C}_{\mathscr{X}, \mathscr{Y} ; h}\right\|_{\mathcal{S}_{\mathcal{U}^{m}, \mathcal{V}^{n}} \leq}^{2} & \frac{3}{\left(\mathscr{L}_{M, N, h}-1\right)^{2}}\left[\mathscr{L}_{M, N, h}^{2}\left\|\hat{m}_{\mathscr{X}}-m_{\mathscr{X}}\right\|_{\mathcal{U}^{m}}^{2}\left\|\hat{m}_{\mathscr{Y}}^{\prime}-m_{\mathscr{Y}}\right\|_{\mathcal{V}^{n}}^{2}\right. \\
& \left.+\mathscr{L}_{M, N, h}^{2}\left\|\hat{\mathscr{C}}_{\mathscr{U}, \mathscr{Y} ; h}-\mathscr{C}_{\mathscr{U}, \mathscr{Y} ; h}\right\|_{\mathcal{S}_{\mathcal{U}^{m}, \mathcal{V}^{n}}}^{2}+\left\|\mathscr{C}_{\mathscr{U}, \mathcal{Y} ; h}\right\|_{\mathcal{S}_{\mathcal{U}^{m}, \mathcal{V}^{n}}}^{2}\right] \\
= & \mathrm{O}_{\mathbb{P}}\left(m n \mathscr{L}_{M, N, h}^{-2}\right)+\mathrm{O}_{\mathbb{P}}\left((1+|h|) m n \mathscr{L}_{M, N, h}^{-1}\right)+\mathrm{O}\left(m n \mathscr{L}_{M, N, h}^{-2}\right) \\
= & \mathrm{O}_{\mathbb{P}}\left((1+|h|) m n \mathscr{L}_{M, N, h}^{-1}\right) .
\end{aligned}
$$

Corollary 3.1. Follows from (3.14), $\|\cdot\|_{\mathcal{L}_{\mathcal{U}^{m}}} \leq\|\cdot\|_{\mathcal{S}_{\mathcal{U}^{m}}}$ and Theorems 3.1-3.2 with $h=0$.

Proof of Lemma 3.2. The assertions are a consequence of (3.20) as well as Theorems 3.1-3.2 with $h=0$, where (3.25) and (3.27) also include (3.23). 
Proof of Theorem 3.5. From the definition of $\breve{\mathfrak{c}}_{j}^{\prime \dagger}$ in (3.16) follows

$$
\left\langle\breve{\mathfrak{c}}_{j}^{\prime \dagger}, \mathfrak{c}_{j}\right\rangle_{\mathcal{U}^{m}}\left\langle\hat{\mathfrak{c}}_{j}^{\prime}, \mathfrak{c}_{j}\right\rangle_{\mathcal{U}^{m}}=1-\left\|\breve{\mathfrak{c}}_{j}^{\prime}-\mathfrak{c}_{j}\right\|_{\mathcal{U}^{m}}^{2}+\frac{1}{4}\left\|\breve{\mathfrak{c}}_{j}^{\prime}-\mathfrak{c}_{j}\right\|_{\mathcal{U}^{m}}^{4}+\left\langle\hat{\mathfrak{c}}_{j}^{\prime}, \mathfrak{c}_{j}\right\rangle_{\mathcal{U}^{m}} \sum_{i=1}^{\infty} \frac{\zeta_{i}\left\langle u_{i}, \mathfrak{c}_{j}\right\rangle_{\mathcal{U}^{m}}}{i^{2} M}
$$

where for the last term holds due to independence of given random variables, $\mathbb{E}\left|\left\langle\hat{\mathfrak{c}}_{j}^{\prime}, \mathfrak{c}_{j}\right\rangle_{\mathcal{U}^{m}}\right| \leq 1, \zeta_{i} \sim$ $\mathcal{N}(0,1)$ for all $i$ and the monotone convergence theorem:

$$
\mathbb{E}\left[\left|\left\langle\hat{\mathfrak{c}}_{j}^{\prime}, \mathfrak{c}_{j}\right\rangle_{\mathcal{U}^{m}}\right| \sum_{i=1}^{\infty} \frac{\left|\zeta_{i}\left\langle u_{i}, \mathfrak{c}_{j}\right\rangle_{\mathcal{U}^{m}}\right|}{i^{2} M}\right]=\mathbb{E}\left|\left\langle\hat{\mathfrak{c}}_{j}^{\prime}, \mathfrak{c}_{j}\right\rangle_{\mathcal{U}^{m}}\right| \sum_{i=1}^{\infty} \frac{\left|\left\langle u_{i}, \mathfrak{c}_{j}\right\rangle_{\mathcal{U}^{m}}\right| \mathbb{E}\left|\zeta_{i}\right|}{i^{2} M}=\mathrm{O}\left(M^{-1}\right) .
$$

Thus, with $(3.28)$ and $1-\operatorname{sgn}\left(1+X_{n}\right)=\mathrm{o}\left(a_{n}\right)$ for real-valued processes $\left(X_{n}\right)_{n}$ with $X_{n}=\mathrm{o}_{\mathbb{P}}(1)$ and real-valued zero sequences $\left(a_{n}\right)_{n}$, for any $j$ indeed holds

$$
\begin{aligned}
\left\|\breve{\mathfrak{c}}_{j}^{\prime \ddagger}-\mathfrak{c}_{j}\right\|_{\mathcal{U}^{m}}^{2} & =\left\|\breve{\mathfrak{c}}_{j}^{\prime}-\mathfrak{c}_{j}\right\|_{\mathcal{U}^{m}}^{2}+2\left[1-\operatorname{sgn}\left(\left\langle\breve{\mathfrak{c}}_{j}^{\prime \dagger}, \mathfrak{c}_{j}\right\rangle_{\mathcal{U}^{m}}\left\langle\hat{\mathfrak{c}}_{j}^{\prime}, \mathfrak{c}_{j}\right\rangle_{\mathcal{U}^{m}}\right)\right] \\
& =\mathrm{O}_{\mathbb{P}}\left(\gamma_{j, m}^{2} m^{2} p M^{-1}\right)+2\left[1-\operatorname{sgn}\left(1+\mathrm{O}_{\mathbb{P}}\left(\gamma_{j, m}^{2} m^{2} p M^{-1}\right)+\mathrm{O}_{\mathbb{P}}\left(\gamma_{j, m}^{4} m^{4} p^{2} M^{-2}\right)+\mathrm{O}_{\mathbb{P}}\left(M^{-2}\right)\right)\right] \\
& =\mathrm{O}_{\mathbb{P}}\left(m^{2} p M^{-1}\right) .
\end{aligned}
$$

Similarly, with (3.25), we also obtain

$$
\begin{aligned}
\sup _{j \leq k}\left\|\breve{\mathfrak{c}}_{j}^{\prime \ddagger}-\mathfrak{c}_{j}\right\|_{\mathcal{U}^{m}}^{2} & \leq \sup _{j \leq k}\left\|\breve{\mathfrak{c}}_{j}^{\prime}-\mathfrak{c}_{j}\right\|_{\mathcal{U}^{m}}^{2}+\sup _{j \leq k} 2\left[1-\operatorname{sgn}\left(\left\langle\breve{\mathfrak{c}}_{j}^{\prime \dagger}, \mathfrak{c}_{j}\right\rangle_{\mathcal{U}^{m}}\left\langle\hat{\mathfrak{c}}_{j}^{\prime}, \mathfrak{c}_{j}\right\rangle_{\mathcal{U}^{m}}\right)\right] \\
& =\mathrm{O}_{\mathbb{P}}\left(m^{2} p M^{-1}\right) .
\end{aligned}
$$

Moreover, due to the definition of $\breve{\mathfrak{c}}_{j}^{\dagger}$ in (3.16),

$$
\begin{aligned}
\mathbb{E}\left[1-\left\langle\breve{\mathfrak{c}}_{j}^{\dagger}, \mathfrak{c}_{j}\right\rangle_{\mathcal{U}^{m}}\left\langle\hat{\mathfrak{c}}_{j}, \mathfrak{c}_{j}\right\rangle_{\mathcal{U}^{m}}\right] & \leq \mathbb{E}|| \breve{\mathfrak{c}}_{j}-\mathfrak{c}_{j} \|_{\mathcal{U}^{m}}^{2}+\mathbb{E}\left[\left|\left\langle\hat{\mathfrak{c}}_{j}, \mathfrak{c}_{j}\right\rangle_{\mathcal{U}^{m}}\right| \sum_{i=1}^{\infty} \frac{\left|\zeta_{i}\left\langle u_{i}, \mathfrak{c}_{j}\right\rangle_{\mathcal{U}^{m}}\right|}{i^{2} M}\right] \\
& =\mathrm{O}\left(m^{2} p M^{-1}\right) .
\end{aligned}
$$

Thus, for any $j$ holds due to the definition of $\breve{\mathfrak{c}}_{j}$ and $\breve{\mathfrak{c}}_{j}^{\ddagger}$, and because of (3.26):

$$
\begin{aligned}
\mathbb{E}\left\|\breve{\mathfrak{c}}_{j}^{\ddagger}-\mathfrak{c}_{j}\right\|_{\mathcal{U}^{m}}^{2} & \leq 2 \mathbb{E}\left\|\breve{\mathfrak{c}}_{j}-\mathfrak{c}_{j}\right\|_{\mathcal{U}^{m}}^{2}+2 \mathbb{E}\left(\left[\operatorname{sgn}\left\langle\breve{\mathfrak{c}}_{j}^{\dagger}, \mathfrak{c}_{j}\right\rangle_{\mathcal{U}^{m}}-\operatorname{sgn}\left\langle\hat{\mathfrak{c}}_{j}, \mathfrak{c}_{j}\right\rangle_{\mathcal{U}^{m}}\right]^{2}\right) \\
& \leq \mathrm{O}\left(m^{2} p M^{-1}\right)+4 \mathrm{P}\left(1-\left\langle\breve{\mathfrak{c}}_{j}^{\dagger}, \mathfrak{c}_{j}\right\rangle_{\mathcal{U}^{m}}\left\langle\hat{\mathfrak{c}}_{j}, \mathfrak{c}_{j}\right\rangle_{\mathcal{U}^{m}}>1 / 2\right) \\
& =\mathrm{O}\left(m^{2} p M^{-1}\right) .
\end{aligned}
$$

Hence, (3.30) is verified, and a similar procedure leads with (3.27) to (3.31).

Proof of Lemma 4.1. The definition of $Z_{N}$ and $\tilde{Z}_{N}$ for any $N \in \mathbb{N}$ yields $Z_{N}-\tilde{Z}_{N}=A^{N}\left(Z_{0}-\tilde{Z}_{0}\right)$, and submultiplicity of the operator norm thus

$$
\mathbb{E}\left\|Z_{N}-\tilde{Z}_{N}\right\|_{\mathcal{H}}^{\nu} \leq\|A\|_{\mathcal{L}_{\mathcal{H}}}^{N} \mathbb{E}\left\|Z_{0}-\tilde{Z}_{0}\right\|_{\mathcal{H}}^{\nu}
$$

Since $\left(Z_{k}\right)_{k \in \mathbb{Z}},\left(\tilde{Z}_{k}\right)_{k \in \mathbb{Z}}$ are $L_{\mathcal{H}}^{\nu}$-processes because $\left(\varepsilon_{k}\right)_{k \in \mathbb{Z}}$ is one and due to the definition of $Z_{k}$ and $\tilde{Z}_{k}$ for all $k$, the expected value on the right is finite. Thus, by choosing $\rho=\|A\|_{\mathcal{L}_{\mathcal{H}}}$ and because $\|A\|_{\mathcal{L}_{\mathcal{H}}}<1$, the assertion is proven.

\section{Acknowledgements}

I thank Alexander Meister (University of Rostock), Siegfried Hörmann (Graz University of Technology), Gregory Rice (University of Waterloo), Piotr Kokoszka (Colorado State University) and Dominik Liebl (University of Bonn) for valuable comments, and Leo Evans for proof-reading. 


\section{References}

[1] Allam A. \& Mourid T. (2019) Optimal rate for covariance operator estimators of functional autoregressive processes with random coefficients. J. Multivariate Anal., 169, 130-137. MR3875591

[2] Aue A. \& Klepsch J. (2017) Estimating functional time series by moving average model fitting. arXiv: $1701.00770 \mathrm{v} 1$

[3] Aue A., Norinho D.D. \& Hörmann S. (2015) On the prediction of stationary functional time series. J. Amer. Statist. Assoc., 110(509), 378-392. MR3338510

[4] Boschi T., Di Iorio J., Testa L., Cremona M. A. \& Chiaromonte F. (2020) The shapes of an epidemic: using Functional Data Analysis to characterize COVID-19 in Italy. arXiv: 2008.04700v1

[5] Bosq D. (2000) Linear Processes in Function Spaces. Lecture Notes in Statistics, 149. New York: Springer. MR1783138

[6] Buckner et al. (2004) A unified approach for morphometric and functional data analysis in young, old, and demented adults using automated atlas-based head size normalization: reliability and validation against manual measurement of total intracranial volume. Neuroimage, 23, 724-738.

[7] Caponera A. (2021) SPHARMA approximations for stationary functional time series on the sphere. Stat. Inference Stoch. Process. URL: https://doi.org/10.1007/s11203-021-09244-6

[8] Caponera A. \& Marinucci D. (2021) Asymptotics for spherical functional autoregressions. Ann. Statist., 49(1), 346-369. MR4206681

[9] Chaouch M. (2014) Clustering-based improvement of nonparametric functional time series forecasting: Application to intra-day household-level load curves. IEEE Trans. Smart Grid, 5(1), 411-419.

[10] Chen Y., Koch T., Lim K.G., Xu X. \& Zakiyeva N. (2020) A review study of functional autoregressive models with application to energy forecasting. Wiley interdisciplinary Reviews: Computational Statistics, e1525.

[11] Dunford N. \& Schwartz J.T. (1988) Linear Operators, Part I: General Theory. New York: John Wiley \& Sons Inc. MR1009162

[12] Ferraty F. \& Vieu P. (2006) Nonparametric Functional Data Analysis. New York: Springer. MR2229687

[13] Gao Y., Shang H.L. \& Yang Y. (2019) High-dimensional functional time series forecasting: an application to age-specific mortality rates. J. Multivariate Anal., 170, 232-243. MR3913038

[14] Gohberg I., Goldberg S. \& Kaashoek M.A. (2003). Basic Classes of Linear Operators (1 ed.). Basel: Birkhäuser Verlag. MR2015498

[15] Gubian M., Torreira F., Strik H. \& Boves L. (2009). Functional data analysis as a tool for analyzing speech dynamics. A case study on the french word c'était. Paper from the 10th Annual Conference of the International Speech Communication Association (INTERSPEECH 2009), in Brighton, United Kingdom, September 6-10, 2009, 2199-2202.

[16] Guillas S. (2001) Rates of convergence of autocorrelation estimates for autoregressive Hilbertian processes. Statist. Probab. Lett., 55(3), 281-291. MR1867531

[17] Hael M. A. (2020) Modeling of rainfall variability using functional principal component method: A case study of Taiz region, Yemen. Model. Earth Syst. Environ.

[18] Hashemi M., Zamani A. \& Haghbin H. (2019) Rates of convergence of autocorrelation estimates for periodically correlated autoregressive Hilbertian processes. Statistics, 53(2), 283-300. MR3916630

[19] Horváth L. \& Kokoszka P. (2012). Inference for Functional Data with Applications. New York: Springer. MR2920735

[20] Hörmann S., Horváth L. \& Reeder R. (2013) A Functional Version of the ARCH Model. Econ. Theory, 29(2), 267-288. MR3042756 
[21] Hörmann S. \& Kokoszka P. (2010) Weakly dependent functional data. Ann. Statist., 38, $1845-1884$. MR2662361

[22] Hsing T. \& Eubank R. (2015). Theoretical Foundations of Functional Data Analysis, with an Introduction to Linear Operators. West Sussex: Wiley. MR3379106

[23] Huang S.-F., Guo M. \& Chen M.-R. (2020) Stock market trend prediction using a functional time series approach. Quant. Finance, 20(1), 69-79. MR4040263

[24] Jarry G., Delahaye D., Nicol F. \& Feron E. (2020) Aircraft atypical approach detection using functional principal component analysis. J.A.T.M., 84, p.101787.

[25] Klepsch J., Klüppelberg C. \& Wei T. (2017) Prediction of functional ARMA processes with an application to traffic data. Econom. Stat., 1, 128-149. MR3669993

[26] Kokoszka P. \& Reimherr M. (2013) Asymptotic normality of the principal components of functional time series. Stoch. Process. Appl., 123(5), 1546-1562. MR3027890

[27] Kokoszka P., Rice G. \& Shang H.L. (2017) Inference for the autocovariance of a functional time series under conditional heteroscedasticity. J. Multivariate Anal., 162, 32-50. MR3719333

[28] Kokoszka P., Stoev S. \& Xiong Q. (2019) Principal components analysis of regularly varying functions. Bernoulli, 25(4B), 3864-3882. MR4010975

[29] Kühnert S. (2019) Über funktionale ARCH- und GARCH-Zeitreihen (German) [About functional $\mathrm{ARCH}$ and GARCH processes]. Doctoral Thesis, University of Rostock.

[30] Kühnert S. (2020) Functional ARCH and GARCH models: A Yule-Walker approach. Electron. J. Statist., 14(2), 4321-4360. MR4187136

[31] Ledoux M. \& Talagrand M. (2011) Probability in Banach spaces. Isoperimetry and processes (Reprint of 1 ed.). Classics in Mathematics. Berlin: Springer. MR2814399

[32] Lung T., Peters M.K., Farwig N., Böhning-Gaese K. \& Schaab G. (2012) Combining long-term land cover time series and field observations for spatially explicit predictions on changes in tropical forest biodiversity. Int. J. Remote Sens., 33:1340.

[33] Martínez-Hernández I. \& Genton M. G. (2020) Recent developments in complex and spatially correlated functional data. Braz. J. Probab. Stat., 34(2), 204-229. MR4093256

[34] Mas A. (2007) Weak convergence in the functional autoregressive model. J. Multivariate Anal., 98(6), 1231-1261. MR2326249

[35] Porro-Muñoz D., Silva-Mata F.J., Revilla-Eng A., Talavera-Bustamante I. \& Berretti S. (2014) 3d face recognition by functional data analysis. Iberoamerican Congress on Pattern Recognition, 818826.

[36] Rice G. \& Shum M. (2019) Inference for the lagged cross-covariance operator between functional time series. J. Time Series Anal., 40(5), 665-692. MR3995663

[37] Rice G., Wirjanto T. \& Zhao Y. (2020) Forecasting value at risk with intra-day return curves. Int. J. Forecast., Elsevier, 36(3), 1023-1038.

[38] Ramsay J. O. \& Silverman B.W. (2005) Functional data analysis (2 ed.). Springer Series in Statistics. Springer, New York. MR2168993

[39] Ruiz-Medina M.D. \& Álvarez-Liébana J. (2019) Strongly consistent autoregressive predictors in abstract Banach spaces. J. Multivariate Anal., 170, 186-201. MR3913035

[40] Ruiz-Medina M.D. \& Espejo R.M. (2012) Spatial autoregressive functional plug-in prediction of ocean surface temperature. Stoch. Environ. Res. Risk. Assess., 26, 335-344.

[41] Sarkar S. \& Panaretos V.M. (2021) CovNet: Covariance Networks for Functional Data on Multidimensional Domains. arXiv: 2104.05021v1 
[42] Sen R. \& Klüppelberg C. (2019) Time series of functional data with application to yield curves. Appl. Stoch. Models Bus. Ind., 35(4), 1028-1043. MR3994372

[43] Spangenberg, F. (2013) Strictly stationary solutions of ARMA equations in Banach spaces. J. Multivariate Anal., 121, 127-138. MR3090473

[44] Stout W.F. (1974). Almost Sure Convergence. New York: Academic Press. MR0455094

[45] Tang C., Wang T. \& Zhang P. (2020) Functional data analysis: An application to COVID-19 data in the United States. arXiv: 2009.08363v2

[46] Tian T.S. (2010) Functional data analysis in brain imaging studies. Front. Psychol., 1, 1-11.

[47] Weidmann J. (1980). Linear Operators in Hilbert spaces, Volume 68 of Graduate Texts in Mathematics. Berlin, New York: Springer. MR0566954

[48] Wong R.K.W. \& Zhang X. (2019). Nonparametric operator-regularized covariance function estimation for functional data. Comput. Statist. Data Anal., 131, 131-144. MR3906800

[49] Yu F., Liu L., Yu N., Ji L. \& Qiu D. (2020) A Method of L1-Norm Principal Component Analysis for Functional Data. Symmetry, 12(1):182. 